ARTICLE

Received 1 Oct 2015 | Accepted 8 Nov 2016 | Published 23 Jan 2017

DOI: $10.1038 /$ ncomms13883 OPEN

\title{
Mechanotransductive cascade of Myo-II-dependent mesoderm and endoderm invaginations in embryo gastrulation
}

Démosthène Mitrossilis ${ }^{1}$, Jens-Christian Röper ${ }^{1, \star}$, Damien Le Roy $^{2,3, \star}$, Benjamin Driquez $^{1}$, Aude Michel ${ }^{4}$, Christine Ménager ${ }^{4}$, Gorky Shaw ${ }^{2,3}$, Simon Le Denmat ${ }^{2,3}$, Laurent Ranno ${ }^{2,3}$, Frédéric Dumas-Bouchiat ${ }^{2,3}$, Nora M. Dempsey ${ }^{2,3} \&$ Emmanuel Farge ${ }^{1}$

Animal development consists of a cascade of tissue differentiation and shape change. Associated mechanical signals regulate tissue differentiation. Here we demonstrate that endogenous mechanical cues also trigger biochemical pathways, generating the active morphogenetic movements shaping animal development through a mechanotransductive cascade of Myo-II medio-apical stabilization. To mimic physiological tissue deformation with a cell scale resolution, liposomes containing magnetic nanoparticles are injected into embryonic epithelia and submitted to time-variable forces generated by a linear array of micrometric soft magnets. Periodic magnetically induced deformations quantitatively phenocopy the soft mechanical endogenous snail-dependent apex pulsations, rescue the medio-apical accumulation of Rok, Myo-II and subsequent mesoderm invagination lacking in sna mutants, in a Fog-dependent mechanotransductive process. Mesoderm invagination then activates Myo-II apical accumulation, in a similar Fog-dependent mechanotransductive process, which in turn initiates endoderm invagination. This reveals the existence of a highly dynamic self-inductive cascade of mesoderm and endoderm invaginations, regulated by mechano-induced medio-apical stabilization of Myo-II.

\footnotetext{
${ }^{1}$ Mechanics and Genetics of Embryonic Development group, Institut Curie, PSL Research University, CNRS, UMR168, Inserm, Marie Curie Univ Paris 06, Institut Curie, 11 rue Pierre et Marie Curie, 75005 Paris, France. ${ }^{2}$ Univ. Grenoble Alpes, Inst NEEL, F-38042 Grenoble, France. ${ }^{3}$ CNRS, Inst NEEL, F-38042 Grenoble, France. ${ }^{4}$ Sorbonne Universités, Université Pierre et Marie Curie Univ Paris 06, Laboratoire PHENIX-UMR 8234, 1 Place Jussieu, 75005 Paris, France. * These authors contributed equally to the work. Correspondence and requests for materials should be addressed to E.F. (email: efarge@curie.fr).
} 
B oth biochemical patterning and biomechanical morphogenesis contribute to shaping living organisms during embryonic development. The role of biochemical and genetic information in the development of a functional three-dimensional tissue morphology is beginning to be well understood in different developmental contexts ${ }^{1-9}$. The reverse role of mechanical cues developed by biomechanical morphogenetic movements, in the activation of developmental biochemical pathways involved in the triggering of cell differentiation, is emerging as a key feature of embryonic development regulation ${ }^{10-12}$. Mechanical cues generated by developmental strain deformation were found to activate major developmental differentiation events, including early anterior endoderm and mesoderm specification ${ }^{13,14}$, antero-posterior axis formation ${ }^{15}$, tooth formation ${ }^{16}$ and joint bone differentiation ${ }^{17}$.

Mechanical signalling has also been suggested to trigger the active morphogenetic movements, including mesoderm invagination ${ }^{18}$, folding in legs and wings during organogenesis ${ }^{19}$, primitive streak formation in chick embryos ${ }^{8}$, and cell-cell junction re-enforcement ${ }^{20,21}$ in animal development, as well as meristem morphogenesis and increased growth rate in plant development ${ }^{22,23}$. Strains developed by one morphogenetic movement were also suggested to mechanically influence another, for example, with posterior endoderm invagination promoting vertex resolution during germ-band extension ${ }^{24,25}$. However, the tools used so far to test mechanical triggering of active morphogenetic movements in the context of morphogenetic deficiencies were based on the use of either soft but nonphysiological direct mechanical deformation (such as indentation or pipette aspiration) or of genetically induced physiological deformation, which is not directly mechanical (Myosin-II (MyoII) activation in neighbouring cells). This opened the possibility of a response to non-physiological mechanical strains or to uncontrolled indirect biochemical pathways independent of mechanical strains, for instance of a Myo-II-triggered re-enforcement of cell-cell junctions potentially leading to Myo-II recruitment in neighbouring cells in a purely biochemical process, respectively.

By developing an innovative micro-magnet-based tool capable of producing dynamic physiological direct mechanical strain in vivo with cell scale resolution, here we show mechanical triggering of active morphogenetic movements by the mechanical cues induced by preceding morphogenetic movements. Specifically, apical constriction of mesodermal cells, and the resulting mesoderm invagination, which corresponds to the first morphogenetic movement of early Drosophila embryogenesis, are both demonstrated to be mechanically activated by soft apical pulses of the mesoderm.

In the Drosophila embryo, mesoderm invagination represents the first morphogenetic movement of embryogenesis. This movement is triggered by medio-apical stabilization of Myo-II, leading to the acto-myosin apical constriction of mesoderm apexes that generates tissue bending and folding ${ }^{26,27}$. Apical constriction follows two distinct phases. The first phase is characterized by snail (sna)-dependent uncoordinated unstable constricting pulsations, associated with unstable medio-apical coalescence of Myo-II spots. The second phase, which leads to invagination, is characterized by a twist (twi)-dependent coordinated stable constriction, associated with stable and progressive coalescence of medio-apical Myo-II spots ${ }^{26,28}$ (Fig. 1a). In sna mutants, both the uncoordinated and coordinated phases are absent, as is mesoderm invagination, thus revealing the interplay between sna and twi pathways in the regulation of mesoderm invagination ${ }^{26,28}$. Medio-apical stabilization of Myo-II, collective apical constriction and mesoderm invagination can be mechanically rescued in sna mutants by indenting the mesoderm by a few microns. This stimulates a mechanosensitive pathway, Folded gastrulation (Fog), that is downstream of twist ${ }^{18}$. Such a response to a non-physiological mechanical perturbation suggested that the mechanotransductive mechanical induction of medio-apical stabilization of Myo-II by the physiological mechanical strains resulting from sna-dependent pulsations could regulate the interplay between sna and twi pathways, which in turn leads to mesoderm morphogenesis.

We show that the medio-apical stabilization of Myo-II in early Drosophila embryo mesoderm is mechanotransductively triggered in response to strain developed by sna-dependent pre-existing individual cell apex pulsations. This is demonstrated by mimicking the physiological apical pulsations absent in sna mutants, resulting in the rescue the Fog-dependent medio-apical stabilization of Rho-associated protein kinase (Rok) and Myo-II, and then mesoderm invagination. Pulsations are induced using time varying, cell scale spatially localized mechanical forces generated within sna mutant embryos, by exploiting magnetic interactions between magnetic nanoparticles (MNPs) encapsulated into liposomes loaded within the embryo, and a near-by array of micro-magnets. In addition, we find that mesoderm constriction and folding leads to the stretching of posterior endoderm cells, which in turn activates the apical accumulation of Myo-II required for its invagination, in a Fog-dependent mechanotransductive process.

Embryonic development has long been considered to be the product of an inductive cascade of developmental gene expression $^{29,30}$. Our results reveal the additional existence of a rapid self-inducting mechanotransductive cascade of active morphogenetic events, driving the sequence of apical stabilization of Myo-II of the first two invaginations in the morphogenesis of early Drosophila embryos. The cascade consists of the sna-dependent apex pulsations triggering medio-apical stabilization of Myo-II and mesoderm invagination, which in turn mechanically activates the apical stabilization of Myo-II that triggers posterior endoderm invagination.

\section{Results}

Micro-magnets to mimic sna-dependent mechanical pulsations. During the early stages of Drosophila embryo development, sna-dependent mesoderm cell apex pulsations initiate at the end of mesoderm cell formation ${ }^{31}$. Using the GFP-tagged transmembrane protein Gilgamesh (Spider-GFP), that labels cell plasma membranes, and Myo-II, which labels the light chain of Myo-II (Myo-II-mCherry), we monitored the change in the surface area of the apex of mesoderm cells and the accumulation of apical Myo-II, respectively ${ }^{28}$. The pulsating phase associated with pulsatile unstable submembranar accumulation of Myo- $\mathrm{II}^{28}$, initiated $2 \mathrm{~min}$ before the end of cellularization, which was defined as time 0 for all experiments. This phase was observed to last $3 \mathrm{~min}$ (Fig. 1b,c). We subsequently observed the initiation of the stable constricting phase, associated with medio-apical stabilization of Myo-II, which drives mesoderm invagination $(\text { Fig. } 1 \mathrm{~b}, \mathrm{c})^{28}$. Fourier transform analysis of the pulsating phase showed a spectral signature characterized by a mean amplitude of relative surface area $22 \pm 2 \%$ with a mean period of $126 \pm 22 \mathrm{~s}$ (Figs 1d and 2g). In sna RNA interference (RNAi) injected Spider-GFP Sqh-mCherry embryos, both the pulsating and stably constricting phases are absent, no characteristic period emerges from the spectral signature and there was no apical accumulation of Myo-II (Figs 1e-g and $2 \mathrm{~g})^{28}$. The pulsatile phase was consistently suppressed after injection of the Y27632 inhibitor of Rok that is required for Myo-II activity (Supplementary Fig. 1a) $^{32,33}$.

To test for the triggering of medio-apical stabilization of MyoII by the mechanical strain generated on mesoderm cells during 
a

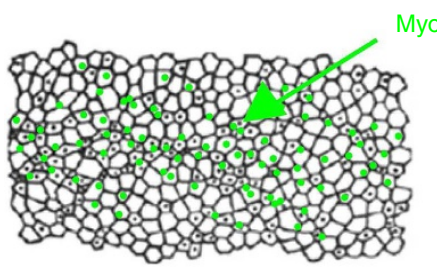

b

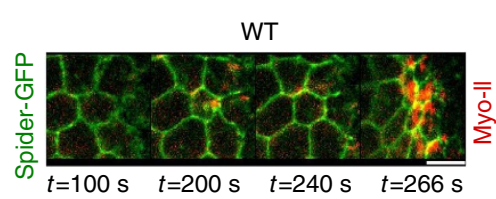

C

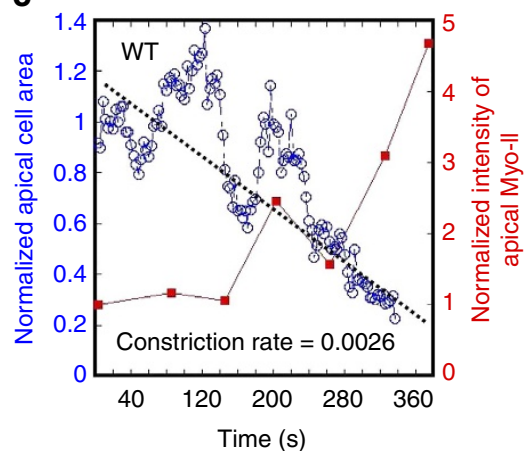

d

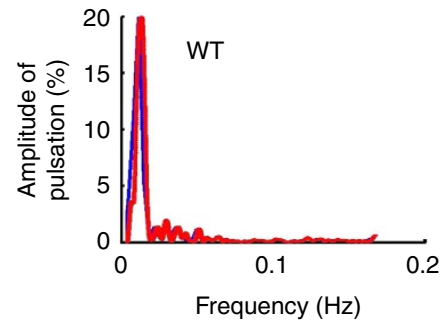

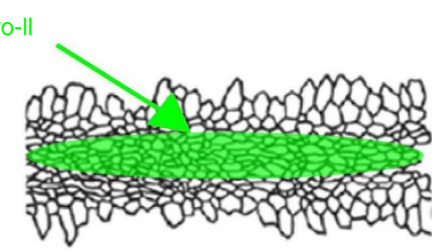

e

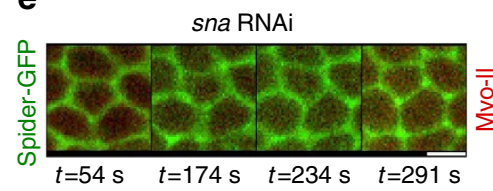

f

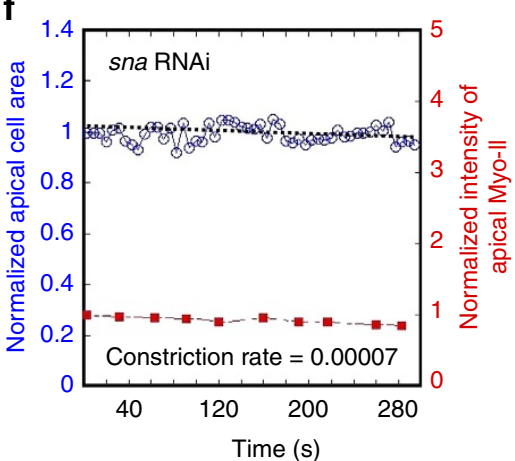

g

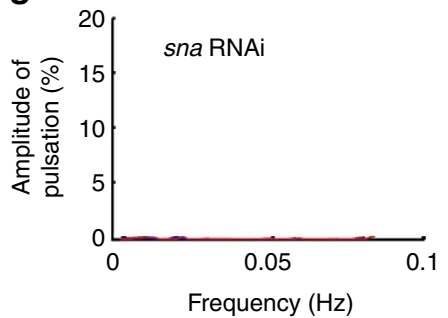

h

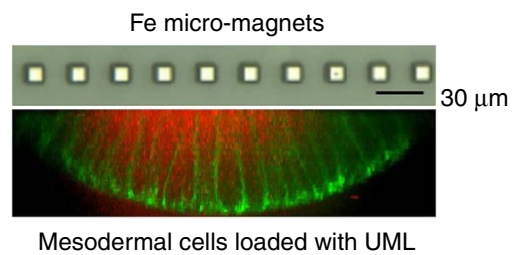

i
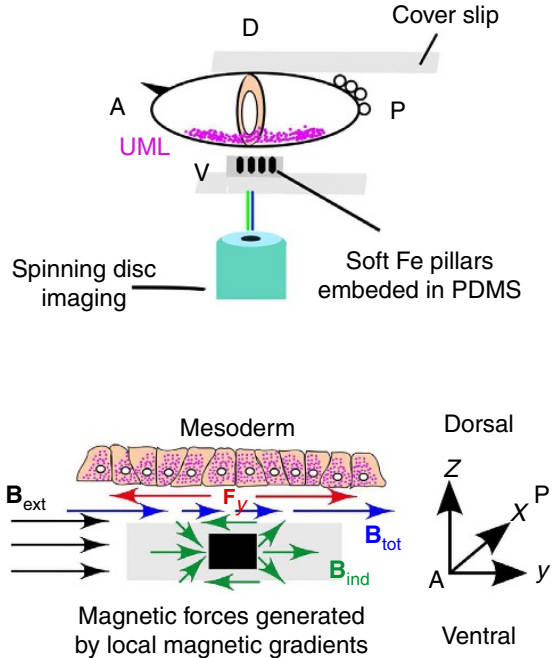

Figure 1 | Use of magnetic forces to mimic sna-dependent apex pulsations in mesoderm cells. (a) Snail-dependent stochastic fluctuation of mesoderm apex size followed by the medio-apical accumulation of Myo-II. (b) Apex size pulsations imaged in the time range 100-240 s, followed by cell constriction (image at 266s) monitored using Spider-GFP (in green), with submembrane apical accumulation of Myo-II (sqh-mCherry in red). (c) Variation of the apical surface area (in blue) and Myo-II concentration (in red) as a function of time in the WT. Representative of the $n=10$ quantified on the $N=64$ pulsating cells, of the 587 mesoderm cells observed, in six distinct embryos. (d) Spectral signature of sna-dependent pulsations in the WT before stable constriction, following Fourier transformation of $\mathbf{c}$. Representative of the $n=8$ cells allowing analysis (that is, staying in the same focus plane long enough) on the $N=64$ pulsating cells of the 587 mesoderm cells observed in six distinct embryos. (e) Absence of cell pulsations, constriction and submembrane apical accumulation of Myo-II, in sna RNAi ( $n=6$ on $N=6$ embryos). (f) Variation of the apical surface area (in blue) and Myo-II concentration (in red) as a function of time in sna RNAi. Representative of the $n=17$ quantified cells of the 892 mesoderm cells observed in six distinct embryos. (g) Spectral signature of sna RNAi, following Fourier transformation of $\mathbf{f}$. (h) Up: optical microscope image (reflection mode) of the micro-magnet network embedded in PDMS. Down: image of fluorescent UML (red) inside the basal domain of mesoderm cells, following the completion of cell formation at the end of stage 5 . Green is Spider-GFP. (i) Up: schematic of the confocal-spinning disk microscope set-up in which the UML-loaded embryo is positioned on top of a transparent PDMS membrane containing a linear array of micro-magnets. Down: schematic of the homogeneous external magnetic field, $\mathbf{B}_{\text {ext }}$ (in black) used to magnetize the soft micro-magnets, producing an inhomogeneous induced field, $\mathbf{B}_{\text {ind }}$ (in green). The gradient of the induced field creates an in-plane tangential repulsive force $F_{y}$ (in red) on UML, magnetized by $\mathbf{B}_{\text {tot }}$ (in blue). A is anterior and $\mathrm{P}$ is posterior. White scale bars are $5 \mu \mathrm{m}$.

sna-dependent pulsations, we developed an innovative approach involving the use of internalized MNPs encapsulated into liposomes and an external array of soft micro-magnets. The soft micro-magnets are magnetized by an external macroscopic magnetic field produced by an electromagnet operated in pulsed mode. When magnetized, the soft micro-magnets produce spatially varying high magnetic field gradients, which serve to apply pulsed forces on superparamagnetic nanoparticles internalized in mesoderm cells (Fig. 1h,i; Supplementary Figs $1 \mathrm{~b}$ and $2 \mathrm{a}$ ). By magnetizing the micro-magnets in a pulsed manner, we effectively apply a pulsed force. The resulting dilating-constricting mechanical forces produced within the cells neighbouring the magnets were used for in vivo quantitative mimicking of apex pulsations.

Specifically, the design of the micro-magnets was guided by the fact that in the wild type (WT), both pulsating cells and first 
a

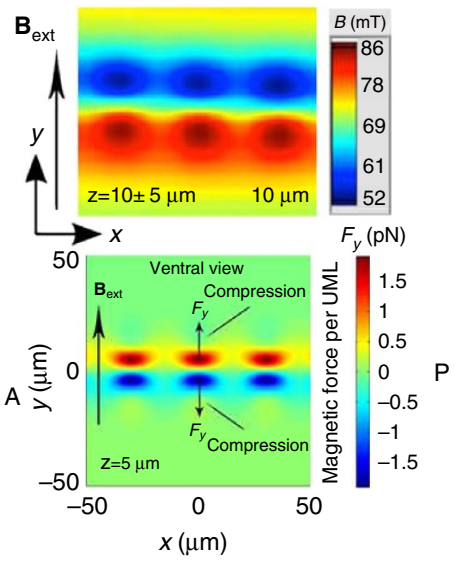

C

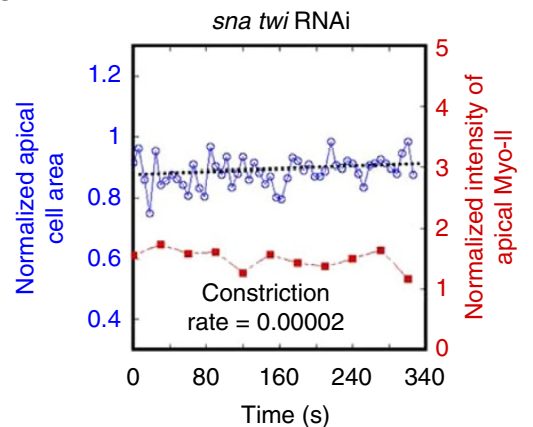

e

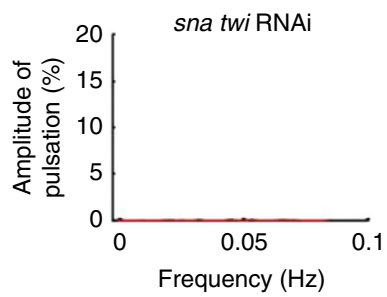

b
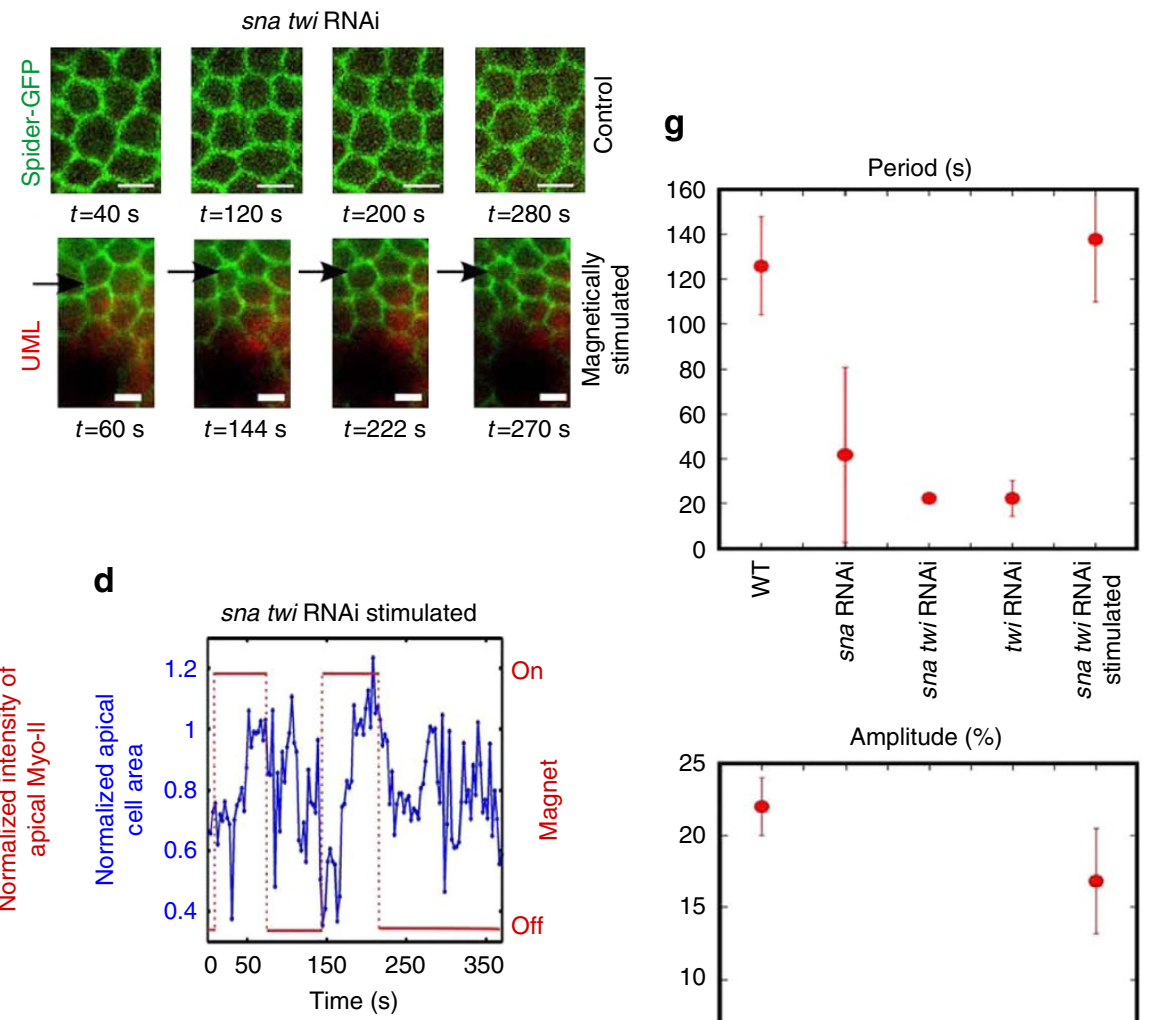

$\mathbf{f}$
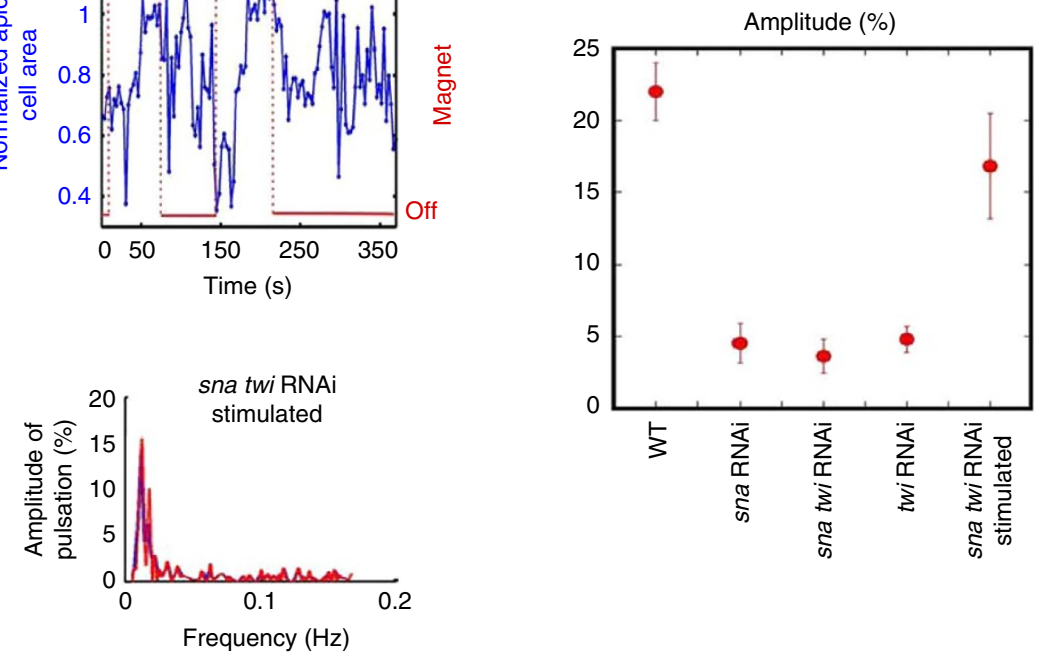

Figure 2 | Mimicking of sna-dependent apex pulsations in mesoderm cells. (a) Up: ventral view of the magnetic field measured on the apical surface of mesoderm cells. Quantitative estimate of the stray field produced in the vicinity of the micro-magnets, measured by scanning hall probe microscopy (probe tilted by $23^{\circ}$ ) at a distance of $10 \pm 5 \mu \mathrm{m}$ above the micro-magnets. Bottom: ventral view of the magnetic force $F_{y}$ on the apex of the mesoderm cells. Simulation of the in-plane ( $y$ component) magnetic force experienced by an UML positioned in the plane $5 \mu \mathrm{m}$ above the micro-magnets. (b) Magnetic stimulation of apex pulsations by the application of a pulsed magnetic field to micro-magnets (shadowy object at bottom of images) positioned close to UML-loaded mesoderm cells on sna twi spider-GFP embryos. Green corresponds to Gilgamesh and red to UML. (c) Variation of the apical surface (in blue) and Myo-II concentration (in red) as a function of time in the sna twi RNAi. Representative of the $n=11$ quantified cells of the $N=745$ cells observed in six distinct embryos. (d) Variation of the apical surface area (in blue) of cell pulsations induced by the application of a pulsed magnetic field to micro-magnets positioned close to UML-loaded mesoderm cells, in sna twi RNAi. Representative of the $n=12$ quantified cells of the 71 pulsating cells on the $N=726$ cells observed in six distinct embryos. (e) Spectral signature of apex surface area fluctuations in the mesoderm cells of sna twi RNAi embryos. Representative of the $n=3$ quantified cells of the $N=745$ cells observed in six distinct embryos. (f) Spectral signature of magnetically induced apex surface pulsations in the mesoderm cells of sna twi RNAi embryos. Representative of the $n=12$ quantified cells of the 55 pulsating cells on the $N=596$ cells observed in five distinct embryos. ( $\left(\right.$ ) Period and amplitude of mesoderm cell apex pulsations in the WT, versus sna RNAi $\left(P_{\text {per }}=0.006, P_{\text {amp }}=0.002\right)$, sna twi RNAi $\left(P_{\text {per }}=0.01\right.$, $\left.P_{\text {amp }}=0.01\right)$, twi RNAi $\left(P_{\text {per }}=0.007, P_{\text {amp }}=0.002\right)$, consistently repressing the pulsating effects of its sna target gene in the presumable case twi RNAi is efficient enough, and sna twi RNAi magnetically stimulated $\left(P_{\text {per }}=0.4, P_{\text {amp }}=0.005\right)$, and sna twi RNAi versus sna twi RNAi magnetically stimulated embryos $\left(P_{\text {per }}=0.013, P_{\text {amp }}=0.011\right)$. $P$ values are calculated from the Mann-Whitney test, and error bars are s.d.

constricting cells are found along the ventral midline of the mesoderm. A linear array of iron micro-magnets, approximately cubic in shape and of edge length $10 \mu \mathrm{m}$, separated by a distance of $20 \mu \mathrm{m}$, was embedded just below the surface of a transparent polydimethylsiloxane (PDMS) membrane of total thickness close to $50 \mu \mathrm{m}$ (Fig. 1i; Supplementary Fig. 2a,b; for fabrication details see the Methods section). Liposomes containing superparamagnetic particles, hereafter referred to as ultra-magnetic liposomes (UMLs, see the Methods section) ${ }^{34}$, were injected into the ventral cells of embryos (Fig. $1 \mathrm{~h}$ and Supplementary Fig. 1b). Once injected embryos were positioned ventrally at a distance of $5 \pm 2 \mu \mathrm{m}$ from the micro-magnets (Fig. 1i), a macroscopic homogenous external magnetic field of $180 \mathrm{mT}$, produced by an electromagnet operated in pulsed ' $60 \mathrm{~s}$ on-60s off mode, was applied in the plane of the micro-magnet array, perpendicular to the linear axis of the array (Supplementary Fig. 2b).

The superparamagnetic particles are subjected to forces induced by the total magnetic field gradient $\nabla\left(B_{\text {tot }}\right)$ on the 
length scale of the micro-magnet size, that is, of the order of tens of microns (Fig. 1i; Supplementary Fig. 2a-d). These forces are proportional to and in the direction of the gradient of the magnetic field amplitude (that is, of the strongest magnetic field $B_{\text {tot }}$ amplitude: $\left.\mathbf{F}=m_{\text {sat }} \nabla\left(B_{\text {tot }}\right)\right)^{35}$, if magnetic moments $m_{\text {sat }}$ are saturated by the magnetic field, which, as a good approximation, is the case everywhere at $z=5 \pm 2 \mu \mathrm{m}$ from the surface of the magnets (Fig. 1i; see Methods).

The external magnetic field produced by the electromagnet $\mathbf{B}_{\text {ext }}$ serves to magnetize the soft iron micro-magnets, thus generating a magnetic dipole hereafter referred to as the induced field $\mathbf{B}_{\text {ind }}$ around each micro-magnet, which leads to the local magnetic field gradients (Fig. 1i; Supplementary Fig. 2c). The existence of this induced local magnetic field gradient was experimentally demonstrated with a scanning hall probe microscope (SHPM), the mean field profile being in quantitative agreement with simulations (Fig. 2a, up; Supplementary Fig. 2d; Methods).

The induced magnetic field $\mathbf{B}_{\text {ind }}$ above and below the micromagnets opposes to the external magnetic field $\mathbf{B}_{\text {ext }}$, and leads to a reduced total magnetic field amplitude of $\mathbf{B}_{\text {tot }}=\mathbf{B}_{\text {ext }}+\mathbf{B}_{\text {ind }}$ compared with the amplitude $\mathbf{B}_{\text {ext }}$ (Fig. 1i; Supplementary Fig. 2c). Therefore, the presence of the pillar generates local gradients of the total magnetic field amplitude $\mathbf{B}_{\text {tot }}$ with smaller values directly above and below the pillar compared with above and below the edges of the pillar (Fig. 1i; Supplementary Fig. 2c, down). The magnetic forces applied to superparamagnetic UML-loaded cells being proportional to, and aligned along the magnetic field gradient, this generates magnetic forces applied on the cells along $y$, orientated from above the pillar to away from the pillar (Fig. 1i). The existence of such repulsive forces is predicted by simulations (Fig. 2a, down; Supplementary Fig. 2e,f). To validate our force simulations, and to demonstrate the existence of repulsive forces in certain regions, we carried out force measurements on a test object $(1.5 \mu \mathrm{m}$ sphere of polystyrene containing superparamagnetic nanoparticles) scanned above the array of micro-magnets, in the presence of an in-plane field (see Methods). Repulsive forces were indeed measured, and good agreement was achieved with simulations (Supplementary Fig. 2g). If the UMLs were free to move, they would follow the force lines and thus move towards the micro-magnets (Figs $1 \mathrm{i}$ and 2a; Supplementary Fig. 2f). UMLs cannot aggregate or move inside a cell, since they are $200 \mathrm{~nm}$ in diameter, and thus trapped within the smaller mesh of the cytoskeleton which generally is of the order of $70 \mathrm{~nm}$ (see Methods) ${ }^{36}$. The magnetic force acting on the cytoskeleton produced by the UML-micro-magnet interactions is thus transmitted by the cytoskeleton in which UMLs are trapped, to the overall cell. Such forces dominate on the most apical part of the cell (see Methods), and thus dilate UML-loaded cells apex below and in front the pillars, while they compress those below and adjacent to the pillars along the dorsoventral $y$ axis, forces being negligible along the anteroposterior $x$-axis (Figs $1 \mathrm{i}$ and $2 \mathrm{a}$ and Supplementary Fig. $2 \mathrm{f}$ ). The application of the external magnetic field in pulsed mode, with a period comparable to the sna-dependent period of apex pulsations (Fig. 1b-g), thus results in soft constriction and dilation pulsatile forces on mesoderm tissue cells in the vicinity of the UML-loaded cells (Figs $1 \mathrm{i}$ and $2 \mathrm{a}$ ). The apical constricting force applied to one UML in one cell was estimated to be of the order of $2 \mathrm{pN}$, leading to a force applied to one cell apex of $0.5 \pm 0.2 \mathrm{nN}$ (Fig. 2a; Supplementary Fig. 2d-g with Methods).

To test our ability to quantitatively mimic snail-dependent pulsations in magnetically loaded embryos using a soft micromagnet array coupled with a pulsating magnetic field, we first studied the ventral midline of sna RNAi twi RNAi-injected Spider-GFP embryos loaded with UML. These lack both active sna-dependent pulsations ${ }^{31}$, and the reactive Fog mechano- sensitive pathway required for apical stabilization of Myo-II, downstream of $t w i^{18}$. In response to the pulsed localized field gradient forces, we observed the generation of individual cell apex size pulsations in the vicinity of the micro-magnets (Fig. 2b; Supplementary Movies 1-3). These pulsations quantitatively mimicked and rescued the sna-dependent pulsations (Fig. 2b-d), with a spectral signature showing a characteristic mean period of $138 \pm 28 \mathrm{~s}$ and a mean amplitude of $17 \pm 4 \%$ (Fig. 2e-g). This signature is comparable to the values of $126 \pm 22 \mathrm{~s}$ and $22 \pm 2 \%$, which are characteristic of sna-dependent pulsations (Figs 1d,g and 2g).

These observations demonstrate the quantitative mimicking of individual cell snail-dependent mechanical pulsations within the multicellular mesoderm of the developing embryo, in a fully biochemically independent process, by the application of pulsating magnetic field gradient forces on UML-injected epithelia.

Mechanical induction of medio-apical stabilization of Myo-II. We then applied pulsating localized magnetic field gradient forces to Spider-GFP; Myo-II-mCherry embryos injected with both blue fluorescent liposomes (Supplementary Fig. 3b) and sna RNAi only, starting 2 min before the end of cellularization, so as to mimic sna-dependent pulsations in the WT.

Focusing on Myo-II-mCherry, we found a medio-apical accumulation of Myo-II, with stable accumulation initiated after a time of $50 \mathrm{~s}$ (Fig. 3a,b; Supplementary Fig. 3a-c), rescued at 74\% of the WT value at $200 \mathrm{~s}$ (Fig. 3c; Supplementary Fig. 4). Such Myo-II accumulation does not occur in non-pulsating sna RNAi-injected embryos, nor in embryos injected with UML and sna RNAi but not treated to magnetic stimulation (Figs 1e,f; Supplementary Fig. 5; Methods), nor in mechanically stimulated sna RNAi twi RNAi-injected embryos (Fig. 2b; Supplementary Fig. 6a,b). Interestingly, one pulse was sufficient to trigger the response in sna RNAi-injected embryos (Supplementary Fig. 6c).

Looking at both the apical submembrane and junctional planes, we observed that magnetic stimulation led to the rescue of stable medio-apical localization of Myo-II, beginning $50 \mathrm{~s}$ after the initiation of stimulation, both in junctions (colocalization with Gilgamesh at cell-cell contact in orange) and medio-apically (in red; Fig. 3e), followed by stable collective constriction (Supplementary Movies 4-6). Both sub-apical junctional and medio-apical submembranar localization of Myo-II mechanical rescue was also observed in halo sna MyoII-GFP mutants (Fig. 3f), in which the halo phenotype allowed us to select homozygous mutants of sna a priori during cellularization and before gastrulation (Supplementary Fig. 1c) ${ }^{28}$.

Focusing on Spider-GFP, we found that magnetic stimulation quantitatively mimicked the first phase of sna-dependent pulsations (Fig. 3b). In contrast to sna RNAi twi RNAi-injected embryos, in which stable collective constriction and invagination were not triggered (Fig. 2d; Supplementary Movies 7 and 8; Supplementary Fig. 6a,b), the pulsatile phase of $150 \pm 12 \mathrm{~s}$, comparable to the $170 \pm 10 \mathrm{~s}$ of the WT, was followed by stable apical constriction of cells, with the constricting rate rescued at nearly $50 \%$ of the WT (Fig. 3b,d). Associated medio-apical accumulation of Myo-II preceded stable apical constriction of mesoderm cells by $100 \pm 12 \mathrm{~s}$ (Fig. 3b), a value also consistent with the $110 \pm 10 \mathrm{~s}$ separating the two events in the WT (Fig. 1c) ${ }^{28}$.

Thus, the magnetically induced quantitative mimicking of the sna-dependent pulsations that rescue medio-apical accumulation of Myo-II in sna embryos, also rescued stable medio-apical constriction and mesoderm invagination from both the sna RNAi and halo sna, sna-defective phenotype (Fig. 4a; Supplementary Movies 4-6 and 9-11; Supplementary Figs 3a-c,e, 7). 
a

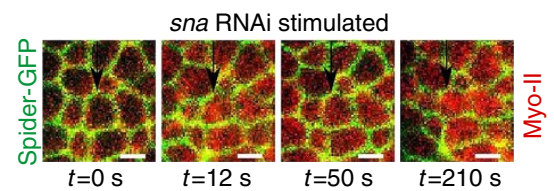

b

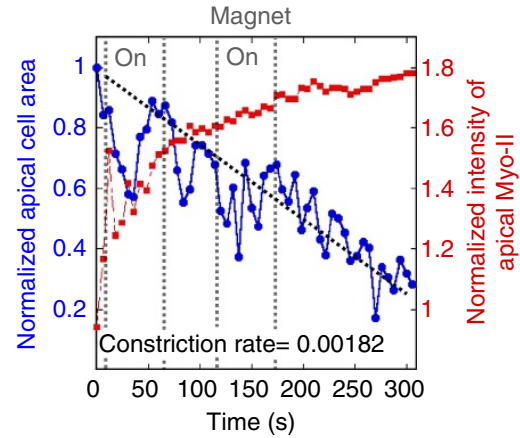

e
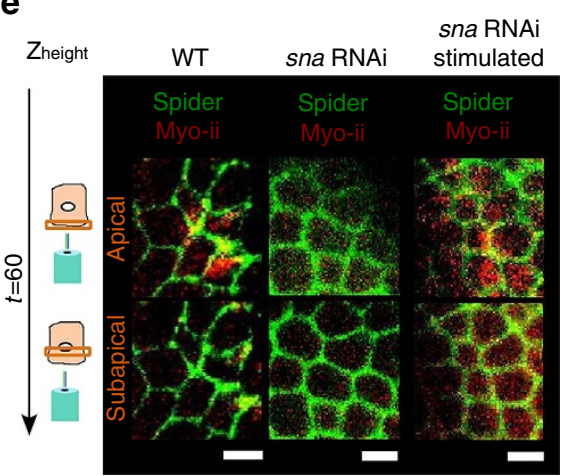

c

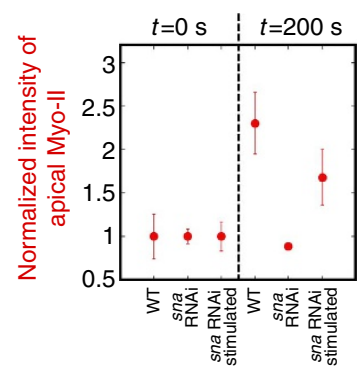

d

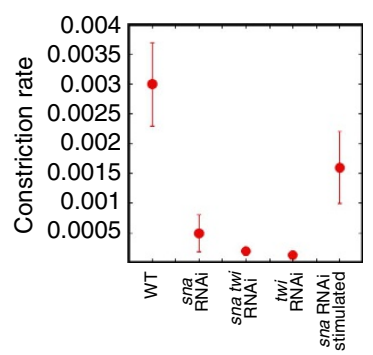

$\mathbf{f}$

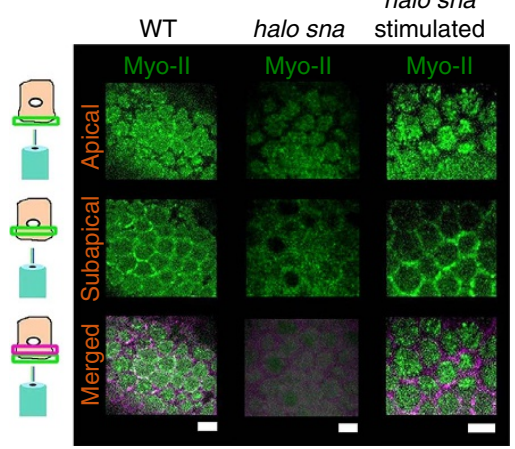

Figure 3 | Rescue of medio-apical Myo-II in sna-defective embryos by mechanical pulses. (a) Dynamical imaging of apex size (Spider, Gilgamesh in green) and accumulation of Myo-II (1 $\mu$ m below, merged in red) in snaRNAi-injected embryos in magnetically stimulated embryos mimicking sna-dependent pulsations in mesoderm cells. Representative of $n=3$ of the $N=5$ embryos ( $P=0.015$, exact Fisher test). (b) Quantification on one representative cell of a. (c) Quantification of the apical accumulation of Myo-II at $200 \mathrm{~s}$ compared with $0 \mathrm{~s}$ in the WT, sna RNAi and sna RNAi magnetically stimulated. $N=6$ embryos for each condition ( $P<10^{-3}$ for both sna RNAi versus WT and sna RNAi magnetically stimulated). (d) Constriction rate in WT ( $N=6$ ), versus sna RNAi-injected $(N=6, P=0.004)$, sna RNAi twi RNAi-injected $(N=6, P=0.02)$, twi RNAi $(P=0.002)$ and sna RNAi magnetically stimulated $(N=4$, $P=0.03$ ) embryos. Scale bars, $5 \mu \mathrm{m}$. $P$ values are calculated from the Mann-Whitney test, and error bars are s.e. (e) Spider (Gilgamesh, in green) and Myo-II (in red) cortical submembrane and junctional apical locations in the mesoderm cells of WT, sna RNAi and sna RNAi magnetically stimulated embryos, at $t=100 \mathrm{~s}$. (f) Myo-II (in green) cortical submembranar and junctional apical locations in the mesoderm cells of WT, halo sna and halo sna magnetically stimulated embryos, at $t=100 \mathrm{~s}$. Representative of $n=8, n=8\left(P<10^{-3}\right.$, exact Fisher test $)$ and $n=6$ embryos $\left(P=7 \times 10^{-3}\right)$ respectively, of the $N=8$ embryos observed in each case.

We then checked, in time lapse in vivo, that a simple mechanical indent of 20 cells by a micromanipulated needle rescued both the apical accumulation of Myo-II and mesoderm cell constriction in snail mutants, initiating at the location of the indent, then propagating all along the mesoderm at a velocity of $0.29 \mathrm{~m} \mathrm{~s}^{-1}$ (Fig. 4b; Supplementary Movie 12). This was confirmed in halo sna mutants (Supplementary Fig. 3d), with a lower efficiency than in the sna indented and in the halo sna magnetically rescued embryos (Fig. 4b Fig. 3f), presumably reflecting the cumulative perturbing effect of the halo genetic mutation and mechanical indent, two non-physiological parameters. Combined with the $150 \mathrm{~s}$ of the morphologic contractile response to magnetic deformation (Fig. $3 \mathrm{~b}$ ), one predicts a range of passive deformation by a minimum of 20 constricting cells initially deformed by the indent able to activate constriction of $0.29 \times 150=45.5 \mu \mathrm{m}$. This represents 8.7 neighbouring cells of $5 \mu \mathrm{m}$ size. In a linear approximation, this predicts that three cells but not two would be able to deform enough the neighbouring cells to trigger the stable constriction of the nearest-neighbour cells.
Such rapid autonomous propagation of active constrictions confirms that apical constrictions are mechanically activated in response to physiologically constricting neighbouring domains, rescued from the snail mutant phenotype.

These results show that the mechanical cues generated by cell pulsations allowed by sna expression are sufficient to trigger the coordinated apical accumulation of Myo-II leading to coordinated apical constriction and to mesoderm invagination.

Medio-apical Rok/Myo-II Fog-dependent mechanical stabilisation. The rescue of apical accumulation of Myo-II leading to constriction and mesoderm invagination by an indent of sna mutants with a needle was suggested to be induced by the mechanical blocking of Fog endocytosis due to membrane straininduced effects, thereby leading to the over-activation of the downstream of the Fog signalling pathway. Indeed, rescue was found to depend on the expression of the Fog protein ${ }^{18}$. Consistent with the fact that Fog is expressed downstream of Twist ${ }^{37}$, we did not rescue apical accumulation of Myo-II, nor collective 
a
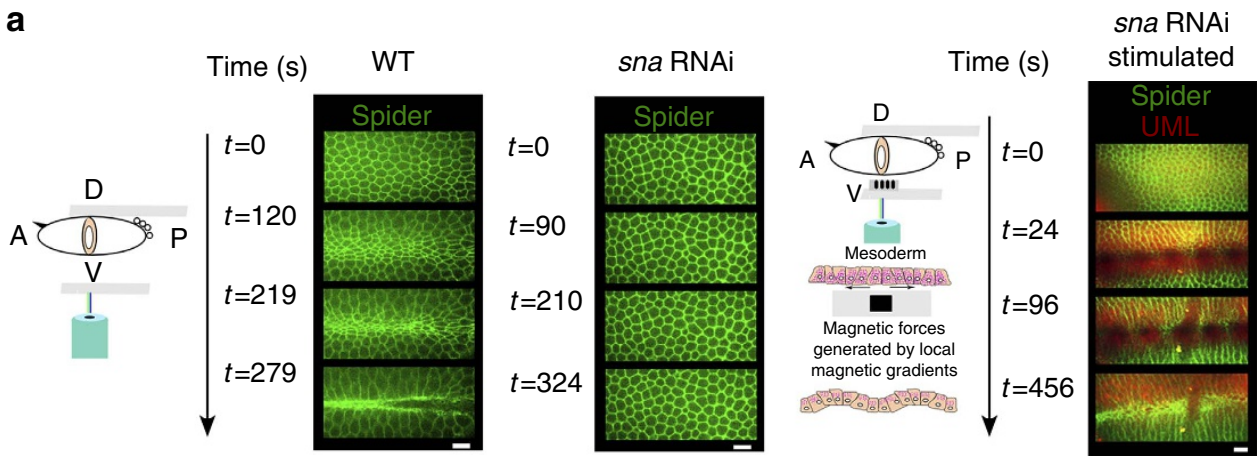

b

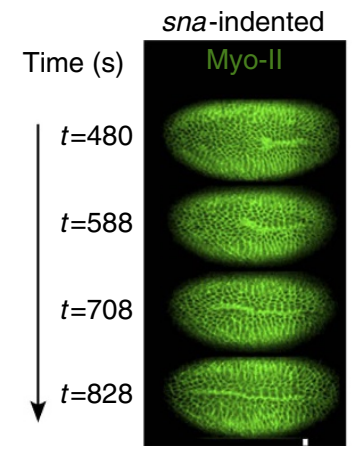

sna- sna-indented
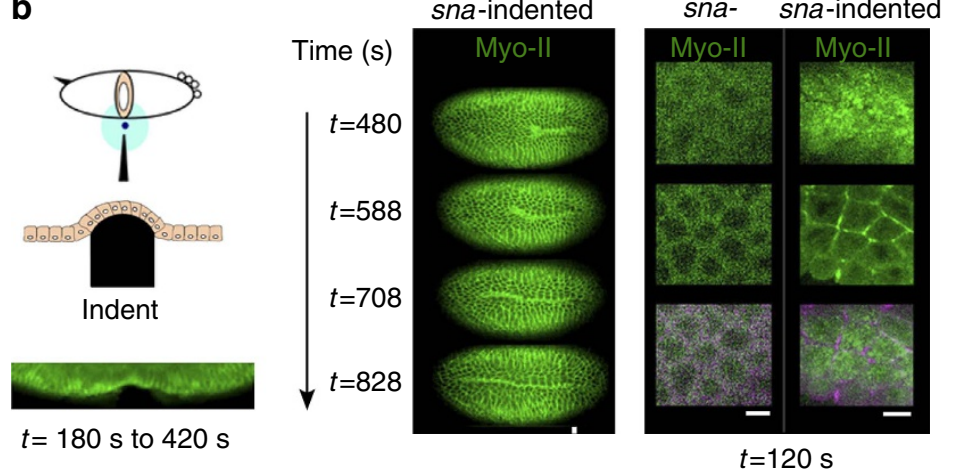

Figure 4 | Rescue of mesoderm invagination in sna-defective embryos by mechanical pulses. (a) Coordinated apical constrictions in Spider-GFP followed by mesoderm invagination in the WT, all of which are impaired in sna RNAi-injected embryos ( $n=6$ on N=6 injected), are rescued (constrictions initiating at $96 \mathrm{~s}$ and mesoderm invagination at $456 \mathrm{~s}$ ) after the initiation of magnetic stimulation of sna-dependent pulsations in sna RNAi-injected embryos (UML in red). Representative of $n=8$ of the $N=13$ embryos observed (full invagination $(n=5)$, partial invagination $(n=3), P<10^{-3}$, exact Fisher test, considering the $N=12$ snaRNAi-injected embryos of Figs 1e and 4 a that do not invaginate). The shadow line is a vitelline membrane folding independent of the process. (b) Mechanical rescue and propagation along the mesoderm of apical stabilization of Myo-Il and mesoderm folding in sna mutant embryos by local indent: sna-Myo-II-GFP embryo indented $3 \mathrm{~min}$ after the end of ventral cellularization, and 4 min before the movie initiation and rotated $90^{\circ}$.

Representative of $n=19$ of the $N=22$ embryos observed (10 total invaginations, 9 partial invaginations, $P<10^{-3}$, exact Fisher test considering that none of the sna mutants invaginate). Zoom on the mesoderm of the indented sna-Myo-II-GFP embryo. Representative of $n=4$ of the $N=4$ embryos observed in each case $\left(P<10^{-3}\right.$, exact Fisher test, with 3 total invaginations, 1 partial invagination). Observations are realized with a $\times 20$ objective. Scale bar, $5 \mu$ m.

constriction nor mesoderm invagination in twiRNAi snaRNAi Myo-GFP embryos submitted to micro-magnet induced mechanical stimulation mimicking sna-dependent pulsations (Fig. 2b,d). Only a partial apical constriction deformation of several mesoderm cells was observed due to local pulsatile magnetic stimulations, thus mesoderm invagination did not occur (Supplementary Fig. 6a,b). We then submitted sna-defective embryos injected with Fog RNAi, to the magnetic stimulation that generated sna-dependent mimetic apex pulsations in sna RNAi-injected Myo-GFP UML-loaded embryos. These embryos showed no detectable rescue of apical accumulation of Myo-II (increase of apical Myo-II to $1.04 \pm 0.14$ compared with before stimulation), nor mesoderm cell constriction nor mesoderm invagination observed in both the WT and the stimulated sna RNAi-injected embryos (Fig. 5a-c; Supplementary Movie 13). Similar results were consistently observed with Rok, for which medio-apical relocalization is required for Myo-II medioapical relocalization ${ }^{38}$ (Supplementary Fig. 8a-c; Supplementary Movies 14-17).

These results show that the Fog protein, which is downstream of Twist, is required in the sna-dependent mechanically induced medio-apical polarization of Rok that leads to mechanically triggered medio-apical accumulation of Myo-II, apical constriction and mesoderm invagination.

Mesoderm apex constriction triggers endoderm apical Myo-II. Apical accumulation of Myo-II leading to epithelial invagination and folding was first identified in the posterior endoderm formation of gastrulating Drosophila embryos ${ }^{1}$. Strikingly, while Sna and Fog were found to be required for apical accumulation of Myo-II and proper invagination of the mesoderm, Fog, but not Snail, is expressed in the posterior endoderm ${ }^{39,40}$. On the other hand, stable apical accumulation of Myo-II in the posterior endoderm initiates at $223 \pm 126 \mathrm{~s}$, after contractile stabilization in the mesoderm at $99 \pm 48 \mathrm{~s}$ (Fig. 6a,e). The accumulation is subsequently reinforced during mesoderm folding until mid-invagination, before the initiation of the germ-band extension morphogenetic movement of convergent extension (Fig. 6a,e). This correlates with the initiation of the posterior endoderm cell stretching observed in response to constriction and folding of ventral mesodermal cells ${ }^{41}$ (Fig. 6a,b,d). We thus tested the hypothesis that the mechanical strain dilations developed by mesoderm cell constriction and folding on the posterior endoderm epithelium activates the apical stabilization of Myo-II, in a Fog-dependent process.

We first characterized apical accumulation of Myo-II in posterior endoderm cells in non-invaginating halo sna mutants. In contrast to the WT, we found no ventral stretching of posterior endodermal cells as observed directly from the position of posterior pole germ cells, and confirmed in the kymographs of posterior endodermal cells (Fig. 6b,d). In contrast to the WT, no apical accumulation of Myo-II in endoderm cells was observed up to $t=204 \mathrm{~s}$ (Fig. 6e), that is, during the equivalent time of the WT first stochastic and second collective constriction phases. We 
a

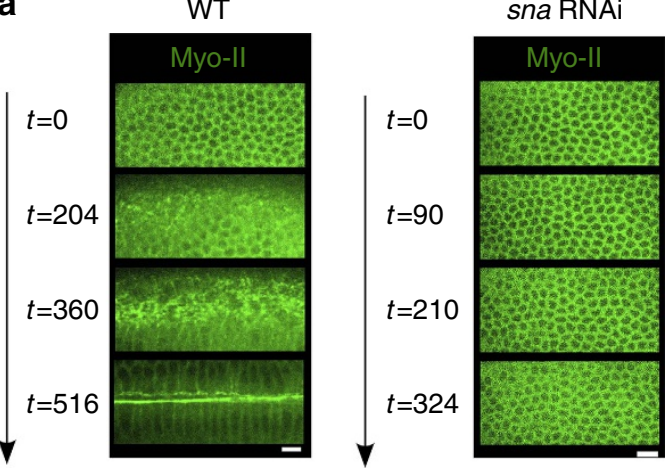

b

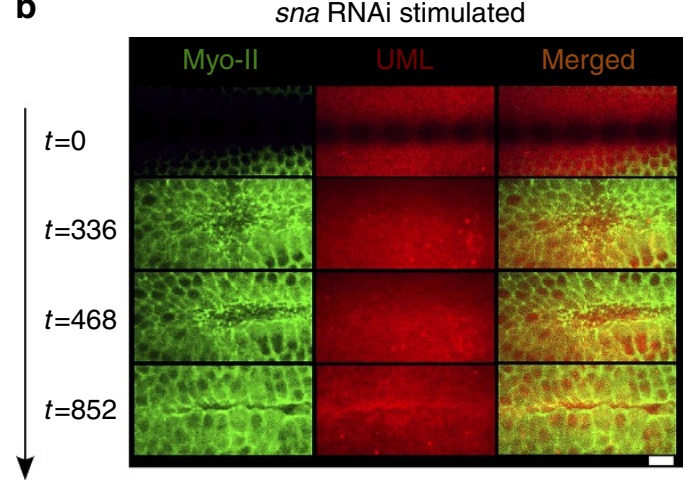

C

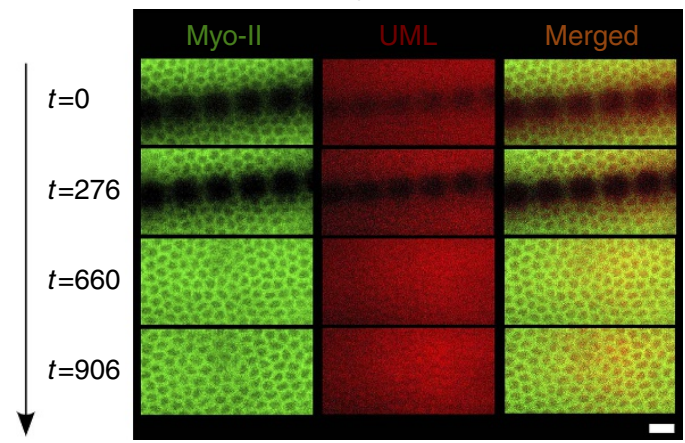

Figure 5 | Rescue of medio-apical Myo-II by sna-like mechanical pulses is Fog-dependent. (a) Myo-II apical accumulation, followed by mesoderm invagination in the WT, all of which are impaired in sna RNAi-injected embryos ( $N=6$ in each case, $P=2 \times 10^{-3}$, exact Fisher test). (b) Apical accumulation of Myo-II and mesoderm invagination after the initiation of magnetic stimulation of sna-dependent pulsations in snaRNAi-injected embryos (Myo-II in green, UML in red). Representative of $n=6$ of the $N=6$ sna RNAi embryos $\left(P=2 \times 10^{-3}\right.$, exact Fisher test, Myo-II apical accumulation $(n=6)$, full invagination $(n=4)$, partial invagination $(n=0)$ ). (c) Sqh-GFP UML-loaded embryos injected with Sna RNAi and Fog RNAi and positioned close to the micro-magnet array, the ensemble being submitted to a magnetic field that is pulsed at a rate that mimics the rate of sna-dependent pulsations. Representative of $n=7$ of $N=8$ embryos $\left(P=5 \times 10^{-3}\right.$, exact Fisher test). The micro-magnet array was removed after $336 \mathrm{~s}$, so as to image the mesoderm directly above the individual micro-magnets. Scale bars, $10 \mu \mathrm{m}$.

rescued the mechanical effect of stretching of posterior endoderm cells by mesoderm invagination in the halo sna mutant, by attracting UML-loaded posterior mesoderm cells with an array of hard (that is, permanently magnetized) micro-magnets of size $20 \mu \mathrm{m}$ deposited onto a patterned Si substrate ${ }^{42}$, attached to a micro-manipulator, $6 \mathrm{~min}$ before the end of cellularization
(Fig. 6c,d). We observed a rescue of the apical accumulation of Myo-II in both the ventral and dorsal endoderm beginning at $t=12 \mathrm{~s}$, namely, $4 \mathrm{~min}$ after the initiation of stretching, and this was re-enforced between $t=204 \mathrm{~s}$ until $t=348 \mathrm{~s}$ (Fig. 6e). The same dynamics of rescue were observed when the stretching was generated from the dorsal side of the embryo, that is, independently of any rescue of mesoderm invagination by magnetic manipulation in the mesoderm, thereby excluding any mechanical-independent developmental rate effect associated to mesoderm formation per se (Supplementary Fig. 9). Given the nearly 3 min delay between the initiation of the magnetic force in the mesoderm and the observation of deformation in the posterior endoderm (Fig. 6d), the timescale of Myo-II apical stabilization rescue is nearly $1 \mathrm{~min}$. This is consistent with the observation of a response initiating nearly $1 \mathrm{~min}$ after the first appearance of apical myosin in the mesoderm of WT (Fig. 6a,e), as well as with the $1 \mathrm{~min}$ timescale of the Myo-II response to magnetically induced and the $<2$ min timescale indent induced, mechanical strains observed in the mesoderm (Figs $3 \mathrm{~b}$ and $4 \mathrm{~b}$, right, respectively). Such rescue was impaired in embryos previously injected with Fog RNAi (Fig. 6e).

Germ-band extension (GBE), which compresses posterior pole cells $8 \mathrm{~min}$ after they have been stretched by mesoderm invagination (Fig. 6a), could subsequently also participate in the posterior endoderm apical accumulation of Myo-II and invagination in a sna-independent process, after stretching due to mesoderm invagination. We thus tested the role of the snadependent mesoderm invagination induced mechanical activation of apical relocation of Myo-II, in endoderm invagination under $b c d$, nos, tor germ-band defective conditions ${ }^{43}$. In sna and $b c d$, nos, tor RNAi-injected embryos, lacking both mesoderm invagination and germ-band extension, no invagination was observed in the posterior endoderm (Fig. 6f). The magnetically induced mimicking of posterior endoderm cell stretching by snadependent mesoderm invagination rescued shallow cup formation, that is, the initiation of endoderm invagination ${ }^{41}$, in these embryos (Fig. 6f). Consistently, bcd, nos, tor RNAi-injected embryos showed shallow cup formation initiation, associated with sna-dependent mesoderm invagination (Fig. 6f; Supplementary Movies 18 and 19; Supplementary Fig. 10e). Note that here the initiation of endoderm invagination is less pronounced than in the WT, possibly due to the lack of an additional germ-band extension buckling driving force in the $b c d$, nos, tor RNAiinjected embryos. Similarly, $b c d$, nos, tsl germ-band defective mutant embryos are also known to initiate posterior endoderm invagination through a shallow cup formation ${ }^{44}$, as observed here with posterior folding and the cup shaped yolk-epithelium interface, respectively-two red arrows in Fig. 6f. Finally, snadefective embryos showed apical accumulation of Myo-II and the initiation of invagination associated to $b c d$, nos, tor-dependent germ-band extension at later stages after $600 \mathrm{~s}$ (Fig. 7).

These experiments demonstrate the Fog-dependent mechanical induction of posterior endoderm apical stabilization of Myo-II and invagination initiation triggered by the mechanical strains developed by mesoderm constriction and invagination, which is subsequently reinforced by morphogenetic movement of GBE.

\section{Discussion}

The search for the biochemical principles regulating epithelial cell folding leading to tissue invagination is a long-standing challenge in developmental biology ${ }^{30}$. Specific interest has been focused on the endoderm and mesoderm primary invaginations of gastrulation, which correspond to the very first morphogenetic movements of embryogenesis in many distinct species ${ }^{45}$. One of the best genetically characterized morphogenetic 
a

$$
\begin{aligned}
& t=0 \mathrm{~s}(120 \mathrm{~s} \text { before the end of ventral cellularization) WT } \\
& \because: \vdots \vdots \vdots \vdots \quad \vdots \quad \vdots \quad \text { Time }(\mathrm{s}) \\
& \vdots \vdots \vdots \vdots \vdots \vdots \\
& :: \vdots: \vdots \quad 309 \pm 37 \mathrm{~s}=\text { posterior endoderm } \\
& \vdots \vdots \vdots \vdots \vdots \quad \text { shallow cup formation } \\
& :: \quad: \quad 245 \pm 108 \mathrm{~s}=\text { beginning of mesoderm invagination } \\
& \vdots \vdots \vdots 223 \pm 126 \mathrm{~s}=\text { stable medio-apical myosin in the } \\
& :: \quad \text { : posterior endoderm cells } \\
& \text { : : } 146 \pm 31 \mathrm{~s}=\text { beginning of collective constriction } \\
& \text { : } 99 \pm 48 \mathrm{~s}=\text { stabilization of apical myosin in the mesoderm cells } \\
& \text { : } 80 \pm 48 \mathrm{~s}=\text { punctual apical myosin in the posterior endoderm cells } \\
& 14 \pm 30 \mathrm{~s}=1 \mathrm{st} \text { apical myosin appearance in the mesoderm cells }
\end{aligned}
$$

b
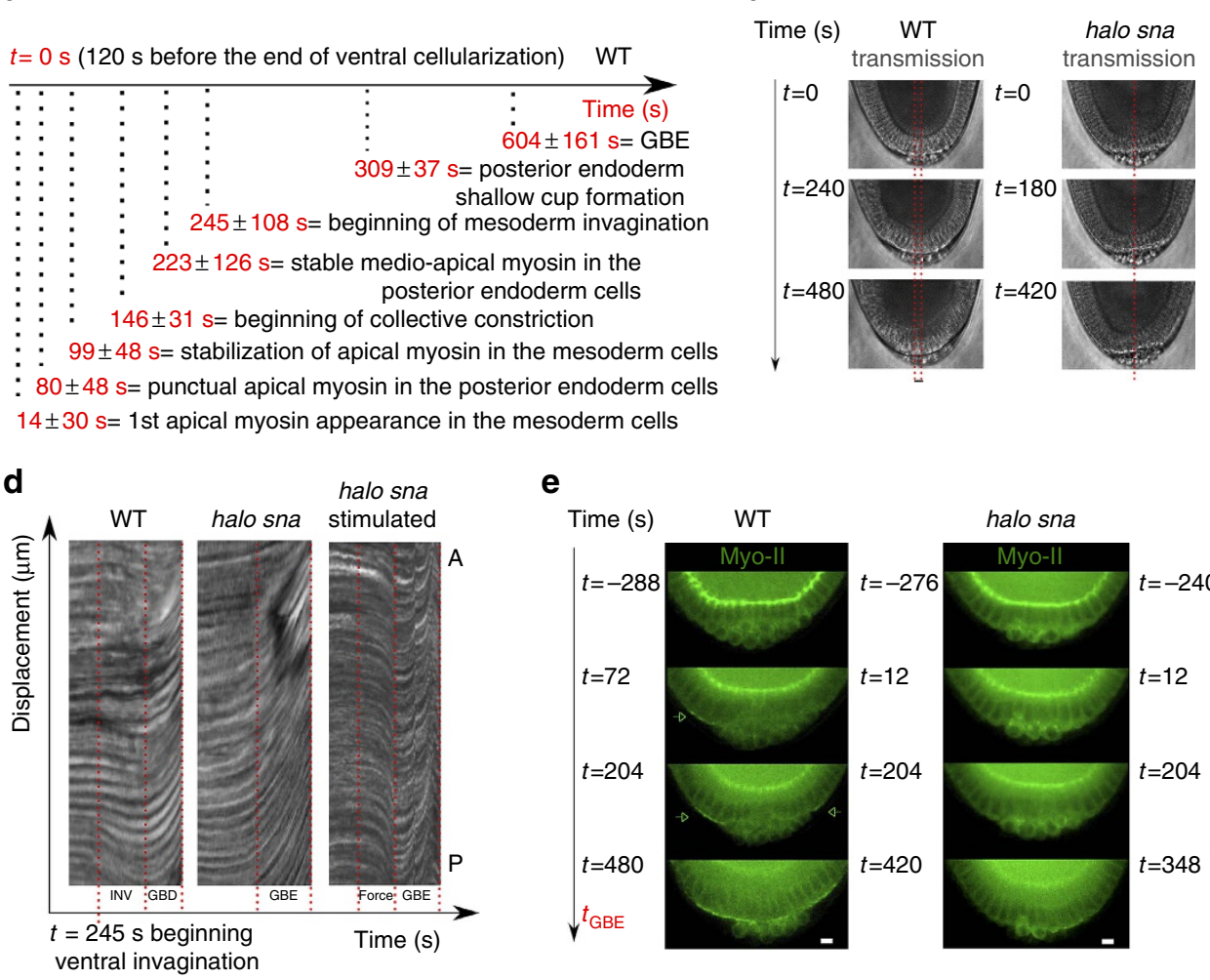

C

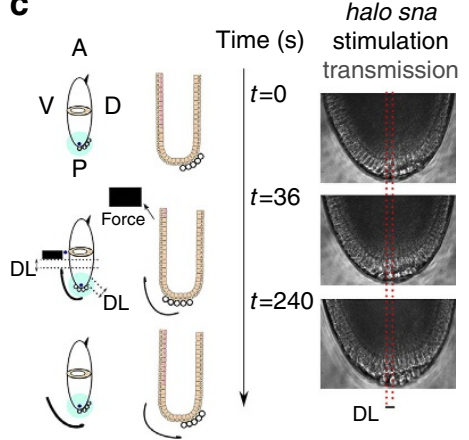

f

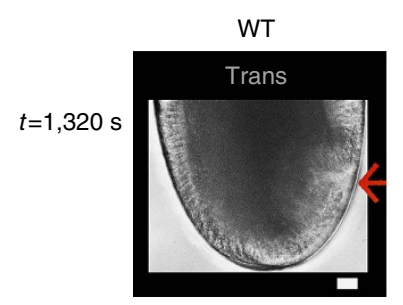

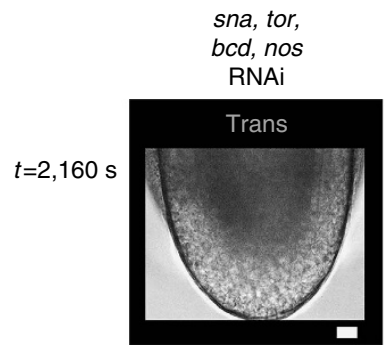

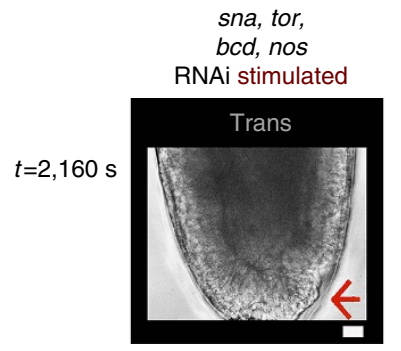

halo sna stimulated

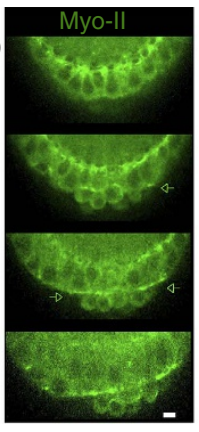

halo sna fog RNAi stimulated

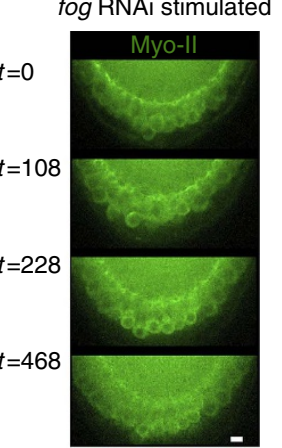

Figure 6 | Fog-dependent induction of endoderm apical Myo-II by mesoderm invagination. (a) Timing of the sequence of morphogenetic events at

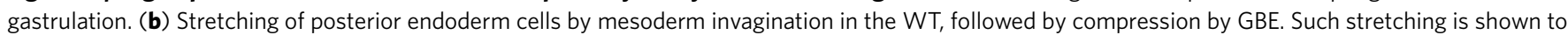
be absent in halo sna mutants. Representative of the $N=6$ embryos observed in each case. (c) Mechanical rescue of posterior endoderm stretching by highly localized magnetic interactions with UML-loaded mesoderm in halo sna mutants. (d) Chemographs of the posterior endoderm of WT, halo sna and magnetically stretched halo sna. Representative of the $N=6$ embryos observed in each case. (e) Distribution of Myo-II-GFP in sqh-GFP, halo sna sqh-GFP, halo sna sqh-GFP magnetically stretched posterior endoderm, and Fog RNAi-injected halo sna sqh-GFP magnetically stretched posterior endoderm. Representative of the $N=6$ WT embryos, the $N=6$ halo sna embryos $P=2 \times 10^{-3}$, exact Fisher test), the $N=14$ halo sna stretched embryos $\left(P<10^{-3}\right.$ ) and the $N=7$ halo sna Fog RNAi-stimulated embryos $\left(P<10^{-3}\right.$ ). Scale bars, $5 \mu \mathrm{m}$. (f) Invagination (red arrow) in the posterior endoderm of non Rnaiinjected (representative of the $N=6$ embryos observed), sna, bcd, nos, tor Rnai-injected (representative of the $N=4$ embryos observed, $P=2 \times 10^{-3}$ ), sna, bcd, nos, tor Rnai magnetically stretched posterior endoderm (representative of $N=5 / 8$ embryos, $P=0.02)$, and $b c d$, nos, tor Rnai embryos $(N=4$, $P=0.03$ ). Scale bars, $10 \mu \mathrm{m}$.

movements is mesoderm invagination of early Drosophila embryos, which is regulated by the interplay between the two pathways established by the expression of the first zygoticaly expressed ventral genes sna and twi. However, while the expression of sna is known to be required for the twidependent stable medio-apical accumulation of Rok and MyoII, mesoderm cell constrictions and invagination, the underlying mechanism of the interplay between the two pathways remains poorly understood $26,31,38,46,47$. Because sna expression leads to cell apex size pulsations through a process that remains to be precisely characterized, thereby introducing mechanical strains in mesoderm cells, it was experimentally (by using a simple but nonphysiological indent mechanical perturbation) and theoretically (by using simulations) suggested that sna could activate the Rho pathway leading to apical accumulation of Myo-II, through a downstream of twi Fog-dependent mechanotransductive process $^{18,48}$. This hypothesis was in line with the finding of a twist-dependent increase of probability of having stable apex constriction in the cells neighbouring already constricting cells ${ }^{49}$.

The results presented above demonstrate that quantitative mimicking of sna-dependent pulsations in the absence of sna expression, leads to (i) the rescue of medio-apical stabilization of Rok, (ii) apical and medio-apical stabilization of Myo-II, (iii) coordinated stable apical constriction and mesoderm invagination. All these processes, absent in sna mutants, are rescued on the timescale of a few minutes. In addition, we show that the rescue of these WT phenotypes from the sna phenotypes is Fogdependent. This demonstrates the existence of a Fog-specific 


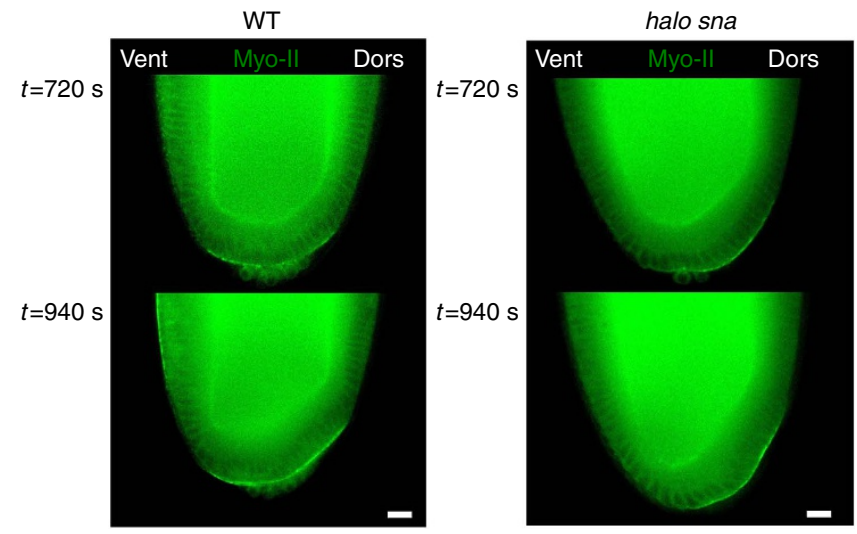

Figure 7 | Distribution of Myo-II-GFP in halo sna at germ-band extension initiation. Representative of the $N=6 \mathrm{WT}$ embryos and the $N=6$ halo sna embryos ( $P=2 \times 10^{-3}$, exact Fisher test). halo sna invaginate their posterior endoderm (later stages, not shown), as do sna mutants. Scale bars, $5 \mu \mathrm{m}$

mechanotransductive activation of medio-apical polarization of Rok, leading to apical stabilization of Myo-II by the mechanical strains resulting from sna-dependent pulsations in the mesodermal cells of Drosophila embryos, on the timescale of a few minutes.

Interestingly, the fact that the rate of constriction rescue is nearly two times lower in the absence of sna than the constriction rate of the WT, and has a lower intensity of Myo-II accumulation at the timescale of invagination initiation ( $>300 \mathrm{~s}$ ), possibly reflects the lack of one of the receptors of Fog (that is, Mist) in sna mutants (Fig. 3b,c) ${ }^{50,51}$. Nevertheless, these less intense responses to mechanically induced pulsations led to mesoderm invagination rescue in sna mutants, with similar dynamics to that of the WT, showing that sna-dependent pulsations are sufficient to trigger the medio-apical stabilization of Myo-II required for mesoderm invagination.

Invagination specifically requires the coordinated constriction of apexes. Coordination of individual cell behaviour can be generated in response to a common external biochemical morphogenetic field, such as the expression of the Fog secreted signal protein ${ }^{46}$. However, ectopic expression of Fog in sna mutants, as well as Fog overexpression in the mesoderm of sna-defective embryos, do not lead to apical accumulation of Myo-II, nor apical constriction nor mesoderm invagination ${ }^{18,39}$. Coordination of individual cell behaviour can be achieved if the behaviour of a given cell activates the same behaviour in its neighbouring cells though cell-cell interactions, thereby leading to correlated collective effects. In the specific case dealt with here, the individual cell behaviour is apical constriction, and is thus mechanical in nature. It was suggested that the mechanical activation of cell constriction by a neighbouring constricting cell can drive collective and coordinated constriction ${ }^{48,52}$. Here we propose that sna-dependent pulsations represent a phase of unstable autonomous cell apex constrictions generating cell-cell mechanical interactions, which trigger a transition to a phase of coordinated apical constriction. Such a transition emerges from a Fog-dependent mechanotransductive activation of the stable constriction of cells mechanically deformed by their neighbouring pulsating constricting cells.

Invagination of both the mesoderm and posterior endoderm is Fog-dependent and induced by a process of apical accumulation of Myo- $\mathrm{II}^{1,41,46}$. Strikingly, we show here that the initiation of both apical Myo-II accumulation in the mesoderm and invagination in the posterior endoderm require the expression of sna (Fig. 6e), while the posterior endoderm does not express sna. However, we found that sna is not biochemically required in itself for the medio-apical accumulation of Myo-II leading to mesoderm invagination, rather the mechanical strains developed by sna-induced pulsations are needed. Similarly, we show that the triggering of apical accumulation of Myo-II and posterior endoderm invagination is induced by the mechanical strain dilations developed by the constriction of mesoderm cells, on the timescale of a few minutes. We also establish the conservation of the Fog-dependent mechanotransductive induction of Myo-II apical accumulation by mesoderm invagination, in the posterior endoderm. Thus, the fact that sna is required to mechanically trigger apical accumulation of Myo-II and mesoderm invagination implies that sna expression is also required to initiate posterior endoderm invagination, even though its expression is not required in posterior endoderm cells. As a consequence, mesodermal sna interacts with posterior endodermal Fog, over the distance of five cells separating the two tissues, through mechanical cues, known to propagate rapidly and over long distances across materials. In addition, we find that mechanical induction of Myo-II apical accumulation and invagination in the posterior endoderm is subsequently reinforced by the morphogenetic movement of GBE.

Embryonic development has long been considered as the product of a cascade of developmental gene expression, with characteristic timescales ranging from $10 \mathrm{~min}$ to hours for each individual step of the cascade 29,30 . Here we demonstrate that the Fog-dependent mechanotransductive induction of medioapical polarization of Myo-II regulates a self-inductive nontranscriptional rapid cascade of active morphogenetic movements at the shorter timescale of minutes. Sna-dependent mesoderm apex pulsations trigger the Fog-dependent medio-apical stabilization of Myo-II that activates the coordinated constriction of apexes generating mesoderm folding and invagination within a few minutes. Such coordinated constriction and mesoderm invagination in turn activates the Fog-dependent apical accumulation of Myo-II initiating the next morphogenetic movement of posterior endoderm invagination, which also occurs within a few minutes (Fig. 8). Snail and Fog expression being the result of the biochemical cascade of differentiation, we thus show the coexistence at different timescales, and interactions between a slower differentiation gene expression biochemical cascade and a rapid self-inductive mechanotransductive cascade. Here we thus reveal that, during and patterned by the expression of developmental differentiation genes, mechanoproteic mechanotransductive interplay drives a rapid selfinducing cascade of morphogenetic movements in early Drosophila gastrulation.

\section{Methods}

Fly strains. Oregon R was taken as the WT. Male and female of any age were used. Myo-II-GFP (sqh-GFP) was provided by Roger Karess, Myo-II-mCherry Gilgamesh-GFP (sqh-mCherry spider-GFP), halo sna Myo-II-GFP (halo snailIIG05/CyO, sqhp-sqh::GFP) were provided by Adam Martin ${ }^{28}$, sna Myo-II GFP (snailIIG05/CyO, sqhp-sqh::GFP) were produced by crossing halo snailIIG05/ CyO, sqhp-sqh::GFP with snailIIG05/CyO, rok-GFP ( $w, U b i-R o k-G F P$; et w; UbiRok-GFP/TM6) ${ }^{21}$ and Gilgamesh-GFP (spider-GFP) ${ }^{53}$ were provided by Yohanns Bellaïche. Stocks were maintained at room temperature $\left(22^{\circ} \mathrm{C}\right)$, and experiments and observations have been done at $24^{\circ} \mathrm{C}$.

RNAi. Three RNAi sequences a gene were designed and produced by Eurogentec Sna RNAi (1,481-1,503 S 5': CAGCUAUAGUAUUCUAAGU dTdT; AS 3': ACUUAGAAUACUAUAGCUG dTdT, 340-362 S 5': GGAACCGAAACGU GACUAU dTdT; AS 3': AUAGUCACGUUUCGGUUCC dTdT, 972-994 S 5': CCGAGUGUAAUCAGGAGAA dTdT; AS 3': UUCUCCUGAUUACACUC GG dTdT).

Twi RNAi (928-950 S 5': GCACCAGCAACAGAUCUAU dTdT; AS 3' AUAGAUCUGUUGCUGGUGC dTdT, 2,061-2,083 S 5': GUCACGCUUUCCA UAUAUA dTdT; AS 3': UAUAUAUGGAAAGCGUGAC dTdT; 1-1,993 S 5': 


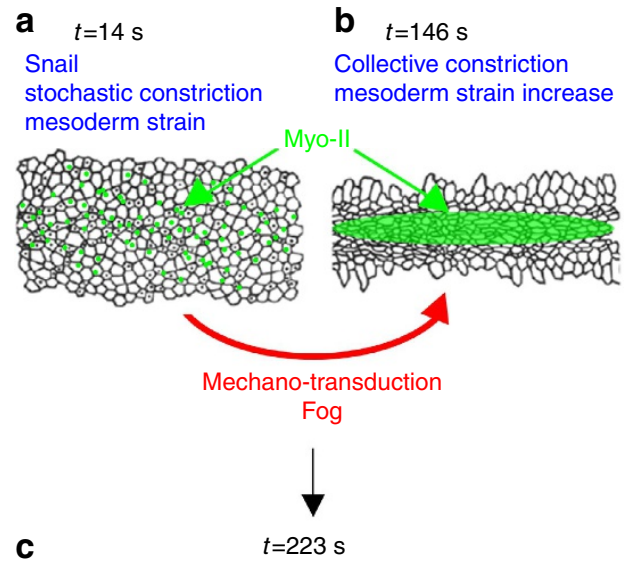

Posterior endoderm strain

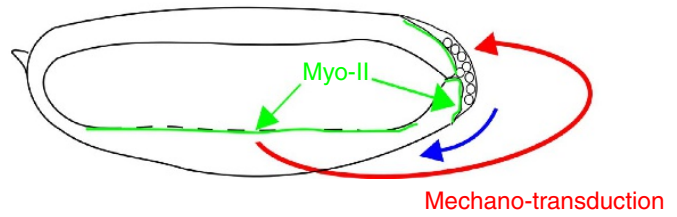

Fog

Figure 8 | Meso-endoderm mechanotransductive cascade of Myo-II apical stabilization. (a) Sna-dependent stochastic fluctuation of mesoderm apex size (b) activates the medio-apical accumulation of Myo-II in a Fog-dependent mechanotransductive process leading to coordinated collective constriction of the cell apex (initiating nearly $130 \mathrm{~s}$ after the initiation of pulsations), then mesoderm invagination. (c) In turn, mesoderm constriction generates a strain deformation of posterior endoderm cells (blue array) that activates the apical stabilization of Myo-II required for endoderm invagination nearly $80 \mathrm{~s}$ after mesoderm collective constriction initiation, that is subsequently reinforced during mesoderm invagination, in a Fog-dependent mechanotransductive pathway. Adapted from the figures of morphogenetic movements of Sweeton et al., ${ }^{26}$ Development, 1991 and Nüslein-Volhard \& Wieschaus, Drosophila a practical approach, 1896.

\section{CGGAUCAGGACACUAUAGU dTdT; AS 3': ACUAUAGUGUCCUGAUCCG} dTdT).

Fog RNAi (98-12,520 S 5': CUGGCCAGAUUAGGUAGAU dTdT; AS 3': AUC UACCUAAUCUGGCCAG dTdT, 7,054-7,076 S 5': CUGAACACCUCACAU AAUA dTdT; AS 3': UAUUAUGUGAGGUGUUCAG dTdT, 3,537-23,559 S 5': CGUGUGAAGUUGAUUUCAA dTdT; AS 3': UUGAAAUCAACUUCAC ACG dTdT).

The sequences were designed to not interfere between themselves, and with myo-II, rhogef2, EB1, cta, T48, traf4, jupiter, arm, src42A, src64B, shg, khc, klc.

Bicoïd-RNAi (3,592-3,614 S 5': CUACUCUACUCGGGUAAAU dTdT, 699-721 S 5': CACACCGACAAUCAGUAAU dTdT, 502-524 S 5': GCGAAAUC UUCUAUUAUCU dTdT).

Nanos-RNAi (1,673-1,695 S 5': GAGCUUCCAAUUCCAGUAA dTdT, 163-185 S 5': GCGAAUACUUCAGUUGAAU dTdT, 726-748 S 5': CGCAGCA GGUAAGAAGAAA dTdT)

Torso-RNAi (771-793 S 5': GUAGUCAUCAAGACCUAAU dTdT, 3,327-3,349 S 5': GUCAGAGCGAUGUGUAAGU dTdT, 2,707-2,729 S 5': CCUCACUCGAAUAACCAAU dTdT).

The effect of RNAi on protein levels was all validated in western blots (see just below), except for Torso, for which no antibody was available in the community (Supplementary Fig. 10a-d).

Western blots. Protein extracts from Drosophila embryos were prepared by collecting dechorionated and injected or Oregon stage 5 embryos and placing them into $40 \mu \mathrm{l}$ lysis buffer $(50 \mathrm{mM}$ Tris $\mathrm{pH} 8.0,150 \mathrm{mM} \mathrm{NaCl}, 0.5 \%$ Triton X-100, $1 \mathrm{~mm}$ $\mathrm{MgCl}_{2}$ with added Complete Protease inhibitor Cocktail without EDTA (Roche) on ice). Embryos were homogenized on ice using a hand homogenizer from VWR with pestle for eppondorf tubes. The homogenate was stored afterwards at $-20^{\circ} \mathrm{C}$ until further use. Homogenate was then centrifuged for $2 \mathrm{~min}$ at 5,000 r.p.m. and $4{ }^{\circ} \mathrm{C}$. A measure of $30 \mu \mathrm{l}$ of the supernatant was stored at $-20^{\circ} \mathrm{C}$. Protein concentration was measured at $280 \mathrm{~nm}$ using the NanoDrop ND-1000 Spectrophotometer system. For western blot sample loading, the equivalent of $10 \mu \mathrm{g}$ was loaded for RNAi-treated embryos and for WT 100. SDS-polyacrylamide gel electrophoresis and blotting were carried out using the Bio-Rad western blot system. Nitrocellulose membranes were blocked using TBST 5\% bovine serum albumin (BSA) for a minimum of $2 \mathrm{~h}$ and then incubated with the primary antibodies overnight in TBST 5\% BSA. Primary antibodies were used in the following dilutions in TBST 5\% BSA: antibodies used were guinea pig a-Sna donated by Eric Wieschaus (Princeton University, USA, dilution 1:2,000) ${ }^{54}$, rabbit a-Twi donated by Siegfried Roth (Köln University, Germany, dilution 1:1,000) ${ }^{55}$, rabbit a-Fog donated by Eric Wieschaus (Princeton University, USA, dilution $1: 400)^{46}$, rabbit a-Nanos donated by Dahua Chen (Beijing University, China, dilution 1:4,000) ${ }^{56}$ and rabbit a-Bicoid donated by Steve Small (New-York University, USA, dilution 1:500 $)^{57}$. Secondary antibodies were horseradish peroxidase-coupled anti-rabbit 1:1,000 (Cell Signaling 7074S) and anti-guinea pig 1:4,000. After incubation of blots with Pierce ECL Plus Western Blotting Substrate (Thermo Scientific) for $5 \mathrm{~min}$, blots were imaged using Amersham Imager 600 . For the lading control, blots were stripped by incubating the blot membrane in SDS $2 \%$, Tris $\mathrm{HCl} \mathrm{pH} 6.863 \mathrm{mM}, 8 \%$ B-mercaptoethanol under the fumehood and slight agitation for $40 \mathrm{~min}$. After washing under running water for $2 \mathrm{~h}$, blots were blocked overnight in TBST 5\% BSA. Primary antibody mouse against $\beta$-actin (AC-15, sc-69879, Santa Cruz Biotechnologies) was added in TBST 5\% BSA $(1: 1,000)$ and incubated overnight. Horseradish peroxidase anti-mouse secondary antibody (Cell Signaling 7076S) was added in a 1:1,000 dilution on TBST. Precise protein molecular weights were calculated from their amino-acid sequence, from the Uniprot database for the annotated proteins (http://www.uniprot.org/): Snail P08044: 42955.33 D; Twist P10627: 54421.83 D; Fog P40795: 76109.92 D; Bicoid P09081: 54511.22 D; Nanos P25724: 43427.78 D; Actin 5c P10987: 41587.51 D. Gel images were analysed using the gel analysis tool in ImageJ, with all bands analysed normalized to respective actin controls.

All experiments were performed on a set of nearly 20 embryos injected at stage 4 , and analysed at cellularization stage, randomly ranging from early stage 5 to late stage 5. The sna RNAi injection induced the specific decrease of $45 \%$ of Sna expression (with no decrease of Bicoid or Nanos expression as a control) as a mean value characteristic of the overall stage 5 (Supplementary Fig. 10a). This was sufficient to lead to the specific inhibition of mesoderm invagination at early stage 6 (Figs 1e,f and 3a). Given the delay between RNA degradation and protein degradation, this is consistent with the $60 \%$ decrease of sna RNA sufficient to inhibit apex pulsations and mesoderm invagination observed in reference ${ }^{28}$. bicoid-RNAi nanos-RNAi torso-RNAi injection induced the specific decrease of $34 \%$ of Bicoid and $34 \%$ of Nanos (with no significant decrease of Twist expression as a control; Supplementary Fig. 10b), as mean values characteristic of the overall stage 5 , which together was sufficient to lead to the specific inhibition of germ-band extension at mid-stage 6 (Supplementary Fig. 10c). Note that the Torso antibody was not currently available (in the community) to test for Torso protein.

Twi RNAi induced the specific decrease of $46 \%$ of Twist expression as a mean value of the overall stage 5 (with no decrease of Bicoid expression as a control; Supplementary Fig. 10c), and Fog RNAi induced the specific decrease of $73 \%$ of Fog expression (with no decrease of Bicoid expression as a control; Supplementary Fig. 10d).

Thus, RNAi treatments let to a specific decrease of the order of $70 \%$ of the signalling protein Fog, of $40 \%$ of the targeted proteins Snail and Twist, and of the order of $30 \%$ on the Bicoid and Nanos proteins having a combining effect in the production of germ-band extension, during stage 5. Such quantitative gradation is consistently inversely related to the developmental time at which the RNAs producing the proteins are initially loaded into the embryo, with bicoid and nanos RNAs being maternally loaded from earliest times of embryonic development ${ }^{58,59}$, whereas twist and snail are zygotically expressed from stage 4, before the onset of cellularization $^{27,60}$, and the strong zygotic expression of $f \circ g$ is characteristic of the later late-stage 5 (ref. 39). In addition, these mean values are representative of the overall cellularization stage 5 (that takes $45 \mathrm{~min}$ of development). Therefore, these are probably significantly underestimated compared with the values specifically characterizing embryos at the end of stage 5 that condition early stage 6 functional effects, which necessarily benefits from the largest amount of time for targeted protein degradation in the absence of renewal under RNAi conditions.

Un-cropped western blots can be found in Supplementary Fig. 11.

Embryo preparation. Egg collections were performed on agar cups covered with grape-juice plates. Flies were allowed to lay eggs for $2-4 \mathrm{~h}$ at $25^{\circ} \mathrm{C}$ before the plate was removed. During preparation, embryos were never compressed to avoid any process of mechanotransduction before controlled mechanical stimulation. In all experiments, Drosophila embryos were dechorionated with 50\% bleach until the 'antenna' disappear (nearly 40 s), then washed with water. Embryos were then glued on a coverslip covered with glue (double-sided tape soaked in heptane), ventral side up (for UML injections Fig. 2), ventral side down (for controls, Fourier transform, Figs 1 and 2) and laterally (for indent experiments and magnetic stimulation Figs 4 and 6). In case of injection, embryos were previously dried $7 \mathrm{~min}$ in the presence of salt. A few droplets of Halocarbon oil 27 were placed on the embryo to prevent drying and to allow observation. After injection, an area of $2.5 \mathrm{~cm} \times 1 \mathrm{~cm}$ including the embryo was cut with a diamond point, turned upside down and glued on the piezo-electric system to observe the ventral side in reflection, and to allow controlled relative movements of the embryo with respect 
to the magnetic pattern with $100 \mathrm{~nm}$ resolution. For indent experiments, the coverslip with the glued embryo was put on the microscope stage, and the indenting needle was attached to the piezo-eclectic device.

Ultra-magnetic nano-particle preparation. The synthesis of MNPs is described first. The aqueous suspension of MNPs was prepared using alkaline co-precipitation of $\mathrm{FeCl}_{2}(0.9 \mathrm{~mol})$ and $\mathrm{FeCl}_{3}(1.5 \mathrm{~mol})$ salts, according to Massart's procedure ${ }^{61}$. Superparamagnetic maghemite grains $\left(\gamma-\mathrm{Fe}_{2} \mathrm{O}_{3}\right)$ were obtained by oxidizing $1.3 \mathrm{~mol}$ of magnetite with $1.3 \mathrm{~mol}$ of iron nitrate (boiling solution). After magnetic decantation, 21 of distilled water and $360 \mathrm{ml}$ of $\mathrm{HNO}_{3} 20 \%$ were added to the solution and the mixture was stirred for $10 \mathrm{~min}$. Prepared maghemite nanoparticles were washed several times with acetone $(3 \times 11)$ and ether $(2 \times 500 \mathrm{ml})$ and suspended in water. Size sorting was performed by adding $\mathrm{HNO}_{3}$ $(0.45 \mathrm{M})$ to the suspension followed by magnetic decantation. This operation was repeated with the deposit until suitable particle size was obtained. Sodium citrate $\left(n_{\mathrm{Fe}} / n_{\mathrm{Cit}}=0.13\right)$ was added to the nanoparticles and the mixture was heated at $80^{\circ} \mathrm{C}$ for $30 \mathrm{~min}$ to promote absorption of citrate anions on their surface. Citrated nanoparticles were precipitated in acetone and suspended in water. The volume fraction and average size of the maghemite grains were determined by fitting the magnetization curve of nanoparticles using the Langevin function. Particles of $9 \mathrm{~nm}$ diameter (s.d. $\sigma=0.35$, volume fraction of nanoparticles in the suspension $\varphi=1.9 \%$, specific susceptibility $\chi / \phi$ of 15.5 ) were obtained. For UML preparation, the aqueous medium was removed using a Macrosep centrifugal device $30 \mathrm{kDa}$ (PALL) and nanoparticles were suspended again in a buffer $(0.108 \mathrm{M} \mathrm{NaCl}, 0.02 \mathrm{M}$ sodium citrate and $0.01 \mathrm{M}$ HEPES, $\mathrm{pH}=7.4$ ).

The preparation of UMLs is described second. Solutions of 1,2-dipalmitoyl$s n$-glycero-3-phosphocholine (DPPC), 1,2-distearoyl-sn-glycero-3-phosphocholine (DSPC), 1,2-distearoyl-sn-glycero-3-phosphoethanolamine-n-[(carboxy (polyethyleneglycol)2000] (ammonium salt) (DSPE-PEG2000) and L- $\alpha$ phosphatidylethanolamine- $N$-(lissamine rhodamine sulfonyl B) (ammonium salt) (Rhod-PE) in chloroform were purchased from Avanti Polar lipids, Inc. UMLs were prepared by the reverse-phase evaporation method established by Skoza et al. ${ }^{62}$ and modified according to a protocol previously described ${ }^{34}$. In brief, a mixture of DPPC/DSPC/Rhod-PE/DSPE-PEG2000 (84/10/1/5 mol\%, $315 \mu \mathrm{l})$ was dissolved in $3 \mathrm{ml}$ of diethyl ether (VWR) and $900 \mu \mathrm{l}$ of chloroform (Carlo Erba Reagents). Thereafter, $1 \mathrm{ml}$ of citrated MNPs dispersed in the buffer was introduced before sonication at room temperature for $20 \mathrm{~min}$ to produce a water-in-oil emulsion. Organic solvent was evaporated with a rotavapor R-210 (Buchi) at $25^{\circ} \mathrm{C}$ until the gel phase disappeared. Liposomes were filtrated through a $450 \mathrm{~nm}$ filter and purified from nonencapsulated MNPs by magnetic sorting using a bulk magnet (Calamit $\mathrm{Fe}-\mathrm{Nd}-\mathrm{B} 150 \times 100 \times 25 \mathrm{~mm}$ ). The operation was repeated three times every $2 \mathrm{~h}$ and liposomes were finally separated from the supernatant and recovered.

Injection of UML and RNAi. An injector (Eppendorf Femtojet) was fixed on the microscope to inject magnetic particles or/and RNAi. A $x, y, z$ piezo-electric system (Princeton Instruments) was mounted on the microscope to control the position of the embryo mounted on a coverslip relative to the injecting needles, the micromagnets or the indent needle, with $100 \mathrm{~nm}$ resolution.

Using a Femtojet Eppendorf injector, $\sim 0.05 \mu \mathrm{l}$ of $0.1 \mu \mathrm{mg}^{-1} \mathrm{UML}$ was injected into embryos along cell basal domain inside the ventral cells of the embryo from 40 to $20 \mathrm{~min}$ before the end of ventral cellularization. Injections were performed at constant pressure, with the visualization of UML labelled with Rhodamin observed in red in spinning disc. Before initiation of the sna-dependent pulsating phase, all blastoderm cells are under cellularization and are basally open to the yolk ${ }^{41}$. UMLs, composed of a high concentration of $9 \mathrm{~nm}$ Fe-oxide superparamagnetic nanoparticles $(20 \% \mathrm{v}-\mathrm{v})$ encapsulated in $200 \mathrm{~nm}$ fluorescently labelled phospholipid membranes ${ }^{34}$, were thus injected into the yolk below and all along mesoderm cells, $20-40$ min before the end of cellularization (Fig. $1 \mathrm{~h}$; Supplementary Fig. 2a). At the end of cellularization, the embryo was turned $180^{\circ}$ ventral to non-magnetized (that is, no external magnetic field applied) soft micro-magnets embedded in a $50 \mu \mathrm{m}$-thick transparent PDMS membrane (Fig. 1i; Supplementary Fig. 2a,b). Note that WT embryos injected with UML gastrulated normally (Supplementary Fig. 1b).

Approximately $0.05 \mu \mathrm{l}$ of $0.1 \mu \mathrm{mg}^{-1} \mathrm{UML}$ and $\sim 0.05 \mu \mathrm{l}$ of $0.1 \mu \mathrm{g} \mu \mathrm{l}^{-1} \mathrm{RNAi}$ were injected from 80 to $60 \mathrm{~min}$ before the end of ventral cell cellularization except for injections including $b c d$, nos and tor Rnai having been injected as soon as possible, $2 \mathrm{~h} 30 \mathrm{~min}$ to $3 \mathrm{~h}$ before the end of cellularization. The UML and the RNAi were injected together inside the embryo along cell basal domain inside the ventral cells. In case of injection of two types of RNAi, embryos were injected from 100 to $80 \mathrm{~min}$ before the end of ventral cell cellularization.

Fabrication and simulation of micro-magnets. Deep reactive ion etching was used to produce a linear array of Si pillars of cross section $10 \times 10 \mu \mathrm{m}^{2}$ and height $40 \mu \mathrm{m}$, separated by a distance of $20 \mu \mathrm{m}$, at the surface of a Si substrate. High-rate triode sputtering was then used to deposit a Ta $(100 \mathrm{~nm}) / \mathrm{Fe}(10 \mu \mathrm{m}) / \mathrm{Ta}(100 \mathrm{~nm})$ trilayer onto the patterned Si substrate (the Ta buffer and capping layers serve to protect the Fe layer from reaction with the substrate and from oxidation, respectively). The thickness of Fe deposited on the sidewalls of the pillars is limited due to the directional nature of sputtering and to shadowing effects from neighbouring pillars. A $50 \mu \mathrm{m}$-thick layer of PDMS was spin-coated onto the structure and, after reticulation, the PDMS layer was pulled off the substrate in such a way that the embedded magnet-topped Si pillars break away from the substrate. The resultant structure consists of a PDMS membrane containing a linear array of soft micromagnets of approximate cubic shape, just below the membrane surface. More details can be found in ref. 63 .

A SHPM was used to measure the stray field pattern produced by the micromagnets ${ }^{64}$. The micro-magnet array was positioned on top of a bulk NdFeB magnet, oriented in such a way as to produce a field that is comparable to that applied by the electromagnet during bio-experiments (in-plane, oriented perpendicular to the axis of the array of micro-magnets). The Hall probe was mounted at an angle of $\sim 23^{\circ}$ with respect to the $y$ axis, for experimental reasons, so that the measured signal had contributions from both the $z$ and $y$ field components $\left(B_{z} \cos 23^{\circ}+B_{y} \sin 23^{\circ}\right)$. The scan was made at a distance of roughly $10 \mu \mathrm{m}$ above the micro-magnet array. Note that the field component measured is determined by the orientation of the probe and that it is not possible to measure the $y$ component of the field pattern above the micro-magnets using our SHPM. Nevertheless, the good agreement observed between measured and simulated patterns validates our simulations of all field components.

Simulation of the stray magnetic field pattern produced by the micro-magnet array was performed. To estimate the force applied by the micro-magnet array on an UML, we need to know the magnetic field produced by it. This was measured using a SHPM, at a scan height of $10 \pm 5 \mu \mathrm{m}$, as shown and described above (Fig. 2a). We then simulated the field profile in an analytical approach using Matlab. We considered a linear array of seven cubes of $\mathrm{Fe}$, of edge length $10 \mu \mathrm{m}$, separated by $20 \mu \mathrm{m}$, magnetized by an in-plane field, applied perpendicular to the axis of the array. The magnetization of the Fe micro-magnets was assumed to be given by the demagnetizing susceptibility so that $M=H_{\text {appl }} / N$, where $N$ is the demagnetizing field factor of the micro-magnet, which is taken to be $1 / 3$. This gives $M=0.18 \times 3=0.54 \mathrm{~T}$. The applied field used in the calculation was that produced by the bulk magnet used to magnetize the micro-magnets in the SHPM. The topologies of the field profiles are comparable, though the peak-to-peak value is higher in the measurement $(34 \mathrm{mT})$ than in the simulation $(18 \mathrm{mT}$; Fig. 2a; Supplementary Fig. 2d). This difference may be attributed to a number of factors, including the non-cubic shape of the real micro-magnets, an error in the estimate of the sample-to-probe distance, and a contribution from the side-wall deposits, neglected in the simulations. Nevertheless, the agreement is considered sufficient to support force simulations shown in Fig. 2a; Supplementary Fig. 2e,g, detailed below.

Quantification of magnetic forces. First, evaluation of forces produced on one UML. The energy of interaction of the UML, with an induced magnetic moment $\mathbf{m}_{\mathrm{UML}}$, and the total magnetic field, $\mathbf{B}_{\mathrm{tot}}$, is: $E=-\mathbf{m}_{\mathrm{UML}} \cdot \mathbf{B}_{\text {tot }}$ (ref. 35). The magnetic force $\mathbf{F}=-\nabla(E)$ is thus: $\mathbf{F}=\nabla\left(\mathbf{m}_{\mathrm{UML}} \cdot \mathbf{B}_{\mathrm{tot}}\right)$. The magnetic moments of the superparamagnetic nanoparticles in the UML are aligned along $\mathbf{B}_{\text {tot }}$ so: $\mathbf{F}=\nabla\left(m_{\mathrm{UML}} B_{\mathrm{tot}}\right)$, where $m_{\mathrm{UML}}$ and $B_{\text {tot }}$ are now scalar. UMLs at $z=5 \pm 2 \mu \mathrm{m}$ above the micro-magnet experience $B_{\text {tot }}$ of $\sim 140 \mathrm{mT}$ (the value is reduced compared to the external field value, because of the opposing induced field) and thus they are almost saturated (see Supplementary Fig. $2 \mathrm{c}$ and the magnetization curve of the UMLs in ref. 34). Thus, $m_{\mathrm{UML}}$ can be considered as almost constant, so that the force applied to a UML is proportional to the gradient of the amplitude of the magnetic field, and is given by: $\mathbf{F}=m_{\mathrm{UML}} \nabla\left(B_{\mathrm{tot}}\right)$. Note that because of the $8 \pm 4 \times 10^{-3}$ concentration of UMLs found in cells after injection (see Evaluation of the number of UML inside mesoderm cells paragraph below), the mean distance between UMLs within cells is thus of the order of 10 times larger than their size $(200 \mathrm{~nm})$, so that attractive interactions between magnetized UML are very small compared with the force of interaction between the UMLs and the micro-magnets, given the $(d / r)^{3}$ decay of the interactions between magnetic moments of magnetic systems of size $d$ at a distance $r$ (ref. 35). What is more, UMLs cannot aggregate, since they are trapped within the mesh of the cytoskeleton which is generally of the order of $70 \mathrm{~nm}$ (ref. 36). Effectively, no apical concentration of UMLs is specifically observed under the magnets after the application of the magnetic field pulses, in the sna twi-defective mutants in which the observation is made easier by the fact that invagination cannot be activated, demonstrating that the UMLs are trapped in the cytoskeleton (Supplementary Fig. 6b, $t=435 \mathrm{~s}$ ). In addition, as confirmed by Supplementary Fig. 1b, UMLs are inside cells, and the UMLs that would remain present out of the cell but within the embryo into the yolk will not significantly contribute to the force applied to the cells, because they are positioned at $>20 \mu \mathrm{m}$ from the magnet, that is, out of the specific range of the application of the force (magnetic forces decay strongly with the distance to the magnet, and practically, only UMLs in the $5 \mu \mathrm{m}$ most apical part of the cell feel significant force, see the first section in the Methods section 'Quantification of magnetic forces' and paragraph below with associated Supplementary Fig. 2f). In addition, there is no extracellular matrix out of the cells into the yolk, and more generally at this stage of development, to trap the UMLs. The magnetic force acting on the cytoskeleton is thus dominated by constricting/dilating forces produced by the UML-micro-magnet interactions, transmitted by the cytoskeleton in which UMLs are trapped, to the overall cell. 
To evaluate the force applied on one UML, let us first consider the saturation values of magnetization and moment. The saturation magnetization of the constituent phase of the MNPs, maghemite, is $M=3 \times 10^{5} \mathrm{Am}=0.38 \mathrm{~T}$. Thus, the saturated moment of a $9 \mathrm{~nm}$ particle is $M V=1.15 \times 10^{-19} \mathrm{Am}^{2}$. Based on magnetic measurements reported in the paper by Bealle et al., the UMLs, of average diameter $200 \mathrm{~nm}$, are filled to roughly 20 vol.\% with $9 \mathrm{~nm}$ diameter MNP. The saturation magnetization of a given UML is thus $76 \mathrm{mT}$, and its saturated moment $2.5 \times 10^{-16} \mathrm{Am}^{2}$. Calculations show that the magnetic field strength at $5 \mu \mathrm{m}$ above the micro-magnet array is $\sim 0.14 \mathrm{~T}$. From the $M(H)$ curves of UML (Supplementary Materials section of ref. 34), $0.14 \mathrm{~T}$ gives $90 \%$ of saturation. From a theoretical point of view, a superparamagnetic spherical MNP has susceptibility limited either by its shape or by its Langevin susceptibility. The spherical MNP used here have a saturation magnetization of $0.38 \mathrm{~T}$ and a demagnetizing factor of $1 / 3$, so the demagnetizing field is $0.38 / 3=0.12 \mathrm{~T}$. If an UML is considered as a homogeneous magnetic sphere with magnetization $20 \%$ that of a MNP (since the UML is $20 \%$ full of MNPs), then its saturation magnetization is $20 \%$ of $0.38 \mathrm{~T}=76 \mathrm{mT}$. Thus, the demagnetizing field of an UML is $25 \mathrm{mT}$. The magnetic moment of a superparamagnetic particle is given by the Langevin function: $m=m_{\mathrm{s}} L(x)$ with $x=m_{\mathrm{s}} \mu_{0} H_{\text {appl }} / k T$. Using $m_{\mathrm{s}}=1.15 \times 10^{-19} \mathrm{Am}^{2}$, $\mu_{0} H_{\text {appl }}=0.18 \mathrm{~T}$ and $T=300 \mathrm{~K}, x=5$ so $m=80 \% m_{\mathrm{s}}$. Thus, we consider that the UMLs and the MNPs are $80 \%$ saturated. $m_{\mathrm{UML}}=2 \times 10^{-16} \mathrm{Am}^{2}$ is used in the force calculations. The force on a UML was calculated as a function of its position with respect to the micro-magnets using analytical expressions $\mathbf{F}=m_{\mathrm{UML}} \nabla\left(B_{t o t}\right)$ (Matlab; Fig. 2a).

Second, measurement and simulation of force on a test object are described. To validate our force simulations, and to demonstrate the existence of repulsive forces in certain regions, we carried out force measurements on a test object scanned above the array of micro-magnets. The test object was a $1.5 \mu \mathrm{m}$ sphere of polystyrene containing 30 volume $\%$ superparamagnetic nanoparticles, supplied by microParticles $\mathrm{GmbH}$. The sphere was stuck to the apex of a commercial atomic force microscope cantilever using a micro-manipulator in a scanning electron microscope. The membrane containing the micro-magnet array was placed on the bulk magnet used for SHPM, so as to apply an in-plane field perpendicular to the axis of the linear array of magnets (that is, along y). The superparamagnetic microsphere was then scanned at different heights above the micro-magnet array, and the deflection of the probe along the $z$ axis was measured as a function of position in the $x-y$ plane (note that the probe was not operated in resonant mode). The level of deflection is directly proportional to the $z$ component of the force exerted by the micro-magnets on the microsphere. As can be seen in Supplementary Fig. 2g, the sign of the force changes from attractive to repulsive, depending on the position of the sphere. The sphere is repelled when it is above the micro-magnets but attracted when displaced along the $y$ axis, in the direction of the applied field. The force on the test object was calculated using analytical expressions (Matlab). Relatively good agreement is found between the measured and calculated forces. Good agreement was also found between the measured and calculated $z$ derivative of the peak-to-peak force, as a function of $z$ (Supplementary Fig. 2g). Though the approach used only allows us to measure the $z$ component of the force, not the $y$ component, it nevertheless validates our approach to simulations and demonstrates that both attractive and repulsive forces can be experienced in the geometry used in our bio-experiments, that is, with an in-plane field serving to magnetize the micro-magnets and the UML within the cells.

To quantify the number of UMLs, we measured the intensity of UML fluorescence per cell, subtracted from the background intensity away from the embryo. This led to local patterns of maximum intensity. Taking the smallest intensity maximum as a single UML signal, we evaluated $18 \pm 7$ UMLs per cell in an optical section $0.5 \mu \mathrm{m}$-thick two-dimensional disc (measured in $N=6$ embryos, 494 cells, error bars being s.d.), namely, $540 \pm 210$ UMLs in a $15 \mu \mathrm{m}$-thick cell of $5 \mu \mathrm{m}$ apical diameter, or an UML concentration of $8 \pm 4 \times 10^{-3}$.

Third, evaluation of forces produced on one cell is described. The force applied apically to UMLs in one cell to compress its neighbouring cell apex by $20 \%$ was thus estimated to be of the order of $0.5 \pm 0.2 \mathrm{nN}$ (considering that $1 / 3$ of the individual UML located in the $5 \mu \mathrm{m}$ most apical part of the cell are submitted to a mean constricting force of $2 \times 10^{-12} \mathrm{~N}$ (Fig. 2a)). This order of magnitude is in line with compression forces of $0.6 \mathrm{nN}$ per cell $(60 \mathrm{nN}$ involved in the compression of $20 \%$ of nearly 100 full cells) ${ }^{65}$.

Note that the volume of the $3 \mu \mathrm{m}$ diameter nucleus approximated as a sphere, that apically excludes UMLs, was evaluated to be $\sim 9 \mu \mathrm{m}^{3}$, which remains small compared with the volume of the apical cylinder in which the nucleus is located (diameter $=5 \mu \mathrm{m}$; length $=5 \mu \mathrm{m}$ so volume $=98 \mu \mathrm{m}^{3}$ ), and was thus not taken into consideration in the evaluation.

Consistently, applying a pulsating homogeneous magnetic field either in the absence of the micro-magnet array (Supplementary Fig. 5) or without injecting UMLs, resulted in neither pulsations nor medio-apical accumulation of Myo-II nor apical constriction. This is in agreement with the fact that the UML feel only a field gradient force, and that the micro-magnets are required to locally concentrate the magnetic field so as to produce localized field gradients. Magnetic field gradients also a priori apply a force normal to the tissue, which could is some cases be detected as leading to a maximum of $0.5 \mu \mathrm{m}$ deformation. However, the mechanical induction of the medio-apical accumulation of Myo-II by normal deformation indentation requires a much higher perpendicular deformation of the tissue, of a minimum of $5 \mu \mathrm{m}$ (ref. 18), ruling out any effect of the normal forces developed by the micro-magnets in the activation of the medio-apical accumulation of Myo-II.

Imaging. A spinning disc (Yokogawa) microscope (IX70 Olympus) coupled to a CoolSnap $\mathrm{HQ}^{2}$ camera was used in all experiments using the 405, 491 and $561 \mathrm{~nm}$ length waves, as well as transmission. All settings, including the piezo-electric control of $z$ position for stack production (Princeton Instrument), were controlled by the Metamorph software, all assembled by Roper. Images have been taken with a 40 times water objective (Olympus LUMPLFN40XW) and a 60 times oil objective (Olympus PLAPON60XO1.42). A 20 times air objective (Olympus UIPLSAPO20XO0.85) was also used for the injections and for some other observations.

Note that the $50 \mu \mathrm{m}$-thick PDMS membrane containing the micro-magnets introduces a new optical index medium in between the glass coverslip and the halocarbon oil in which the embryos are immerged, and that diffraction is introduced by the presence of the $10 \mu \mathrm{m}$ iron cubes every $20 \mu \mathrm{m}$. This introduces noise in the images that however does not prevent cell resolution analysis. Note also that the embryos are not flattened against the coverslip or PDMS to avoid any mechanically inducted interference due to the squeezing. Thus invaginations generated large movements that frequently obliged the operator to re-centre the image to not lose the cell apexes. This prevented also the use of $\times 60$ magnification during the constriction phase, to not lose the imaging field and mean focalization plan. The use of $\times 40$ introduced pixelarization in some cases.

Image analysis. Apical cell shapes were imaged at junctions, $3 \mu \mathrm{m}$ in $z$ (in depth) below the apical surface of the epithelial cells. Individual apical cell areas were measured using FIJI and Freed software. Time lapse was realized in vivo every $3 \mathrm{~s}$ using spinning disc imaging of the apical domain of the cells of the ventral side of Drosophila embryos labelled with spider-GFP and sqh-Cherry. Individual cell tracking were realized afterwards, in silico. Individual cells were manually tracked. The observation started $120 \mathrm{~s}$ before the end of ventral cellularization, and ended $300 \mathrm{~s}$ after, at the beginning of germ-band extension. Matlab software was used to execute Fourier transform on the dynamics of individual apex cell shape changes. No filtration was applied to generate the Fourier transform. The drift (mean slope of the basal corresponding to stable constriction that adds to pulsations) was removed, to analyse the signal that is associated to pulses only. Mean values and graphs were realized with Kaleidagraph software.

Time-lapse resolution is of the order of $1 \mathrm{~min}$ for whole mesoderm imaging.

Fluorescent intensity was quantified by subtracting the background intensity (away from the embryo) from the signal measured in cells. Correlation functions between distant pixels were evaluated using FIJI (http://imagejdocu.tudor.lu/doku.php?id=macro:radially_averaged_ autocorrelation; http://rsb.info.nih.gov/ij/plugins/radial-profile.html). Author Paul Baggethun.

Kymographs were realized in transmission, using ImageJ.

Drosophila mechanical indents. A micromanipulated $50 \mu \mathrm{m}$ diameter needle was approached close to the mesoderm of stage 5. In the specific case of sna-/Cyo;sqhGFP embryos. Embryos having not initiated apical accumulation of Myo-II 2 min after the end of ventral cellularization were genotyped as sna homozygous. Embryos were systematically indented by $3-7 \mu \mathrm{m}$ during $4 \mathrm{~min}$ at the most posterior $1 / 3$ position of the mesoderm, 2-3 min after the end of cellularisation. After stimulation, the embryo was turned $90^{\circ}$ for observation of the ventral side.

Statistics. All statistics based on quantitative measurements were evaluated with the non-parametric two-sided Mann-Whitney test, with s.d. error bars being comparable within each group of data, and the variance between groups of data being similar. Error bars are s.d., except in Fig. $3 \mathrm{f}$ in which they are s.e. Most of the experiments were reproduced at least six times in the laboratory, which is standard in single embryo in vivo experiments. $n$ is the number of measurements per experiment. All statistics based on qualitative measurements (on/off results, that is, the presence of an invagination or not) were evaluated with the exact Fisher test.

Data availability. The authors declare that all data supporting the findings of this study are available within the article and its Supplementary Information files or from the corresponding author upon reasonable request.

\section{References}

1. Young, P. E., Pesacreta, T. C. \& Kiehart, D. P. Dynamic changes in the distribution of cytoplasmic myosin during Drosophila embryogenesis. Development 111, 1-14 (1991).

2. Zallen, J. A. \& Wieschaus, E. Patterned gene expression directs bipolar polarity in drosophila. Dev. Cell 6, 343-355 (2004).

3. Bertet, C., Sulak, L. \& Lecuit, T. Myosin-dependent junction remodelling controls planar cell intercalation and axis elongation. Nature 429, 667-671 (2004). 
4. Butler, L. C. et al. Cell shape changes indicate a role for extrinsic tensile forces in Drosophila germ-band extension. Nat. Cell. Biol. 11, 859-864 (2009).

5. Sagner, A. et al. Establishment of global patterns of planar polarity during growth of the Drosophila wing epithelium. Curr. Biol. 22, 1296-1301 (2012).

6. Bosveld, F. et al. Mechanical control of morphogenesis by Fat/Dachsous/Fourjointed planar cell polarity pathway. Science 336, 724-727 (2012).

7. Aliee, M. et al. Physical mechanisms shaping the Drosophila dorsoventral compartment boundary. Curr. Biol. 22, 967-976 (2012).

8. Rozbicki, E. et al. Myosin-II-mediated cell shape changes and cell intercalation contribute to primitive streak formation. Nat. Cell Biol. 17, 397-408 (2015)

9. Naganathan, S. R., Furthauer, S., Nishikawa, M., Julicher, F. \& Grill, S. W. Active torque generation by the actomyosin cell cortex drives left-right symmetry breaking. eLife 3, e04165 (2014).

10. Wozniak, M. \& Chen, C. S. Mechano-tranduction: a growing role for contractibility. Nat. Rev. Mol. Cell Biol. 10, 34-43 (2009).

11. Mammoto, T. \& Ingber, D. E. Mechanical control of tissue and organ development. Development 137, 1407-1420 (2010).

12. Farge, E. Mechanotransduction in development. Curr. Top. Dev. Biol. 95, 243-265 (2011)

13. Desprat, N., Supatto, W., Pouille, P. A., Beaurepaire, E. \& Farge, E. Tissue deformation modulates twist expression to determine anterior midgut differentiation in Drosophila embryos. Dev. Cell. 15, 470-477 (2008).

14. Brunet, T. et al. Evolutionary conservation of early mesoderm specification by mechanotransduction in Bilateria. Nat. Commun. 4, 2821 (2013).

15. Hiramatsu, R. et al. External mechanical cues trigger the establishment of the anterior-posterior axis in early mouse embryos. Dev. Cell 27, 131-144 (2013).

16. Mammoto, T. et al. Mechanochemical control of mesenchymal condensation and embryonic tooth organ formation. Dev. Cell 21, 758-769 (2011).

17. Kahn, J. et al. Muscle contraction is necessary to maintain joint progenitor cell fate. Dev. Cell 16, 734-743 (2009).

18. Pouille, P. A., Ahmadi, P., Brunet, A. C. \& Farge, E. Mechanical signals trigger myosin ii redistribution and mesoderm invagination in Drosophila embryos. Sci. Signal. 2, ral6 (2009).

19. Monier, B. et al. Apico-basal forces exerted by apoptotic cells drive epithelium folding. Nature 12, 245-248 (2015).

20. Fernandez-Gonzalez, R., Simoes Sde, M., Roper, J. C., Eaton, S. \& Zallen, J. A. Myosin II dynamics are regulated by tension in intercalating cells. Dev. Cell 17, 736-743 (2009).

21. Bardet, P. L. et al. PTEN controls junction lengthening and stability during cell rearrangement in epithelial tissue. Dev. Cell 25, 534-546 (2013).

22. Hamant, O. et al. Developmental patterning by mechanical signals in Arabidopsis. Science 322, 1650-1655 (2008).

23. Uyttewaal, M. et al. Mechanical stress acts via katanin to amplify differences in growth rate between adjacent cells in Arabidopsis. Cell 149, 439-451 (2012).

24. Collinet, C., Rauzi, M., Lenne, P. F. \& Lecuit, T. Local and tissue-scale forces drive oriented junction growth during tissue extension. Nat. Cell Biol. 17, 1247-1258 (2015).

25. Lye, C. M. et al. Mechanical coupling between endoderm invagination and axis extension in Drosophila. PLoS Biol. 13, e1002292 (2015).

26. Sweeton, D., Parks, S., Costa, M. \& Wieschaus, E. Gastrulation in Drosophila: the formation of the ventral furrow and posterior midgut invaginations. Development 112, 775-789 (1991).

27. Leptin, M. twist and snail as positive and negative regulators during Drosophila mesoderm development. Genes Dev. 5, 1568-1576 (1991).

28. Martin, A. C., Kaschube, M. \& Wieschaus, E. F. Pulsed contractions of an actinmyosin network drive apical constriction. Nature 457, 495-499 (2009).

29. Spemann, H. \& Mangold, H. Foundations of Experimental Embryology 144-184 (Hafner, 1924).

30. Gilbert, S. F. Developmental Biology 11th edn (Sinauer Associates, 2016)

31. Martin, A. C. Pulsation and stabilization: contractile forces that underlie morphogenesis. Dev. Biol. 341, 114-125 (2009).

32. Wang, Y. et al. ROCK isoform regulation of myosin phosphatase and contractility in vascular smooth muscle cells. Circ. Res. 104, 531-540 (2009).

33. Monier, B., Pelissier-Monier, A., Brand, A. H. \& Sanson, B. An actomyosinbased barrier inhibits cell mixing at compartmental boundaries in Drosophila embryos. Nat. Cell Biol. 12, 60-65 (2010).

34. Bealle, G. et al. Ultra magnetic liposomes for MR imaging, targeting, and hyperthermia. Langmuir 28, 11834-11842 (2012).

35. Feynman, R. P. The Feynman Lectures on Physics (Addison-Wesley Publishing Company, 1964).

36. Etoc, F. et al. Magnetogenetic control of protein gradients inside living cells with high spatial and temporal resolution. Nano. Lett. 15, 3487-3494 (2015).

37. Costa, M., Wilson, E. T. \& Wieschaus, E. A putative cell signal encoded by the folded gastrulation gene coordinates cell shape changes during Drosophila gastrulation. Cell 76, 1075-1089 (1994).
38. Mason, F. M., Tworoger, M. \& Martin, A. C. Apical domain polarization localizes actin-myosin activity to drive ratchet-like apical constriction. Nat. Cell Biol. 15, 926-936 (2013).

39. Morize, P., Christiansen, A. E., Costa, M., Parks, S. \& Wieschaus, E. Hyperactivation of the folded gastrulation pathway induces specific cell shape changes. Development 125, 589-597 (1998).

40. Alberga, A., Boulay, J. L., Kempe, E., Dennefeld, C. \& Haenlin, M. The snail gene required for mesoderm formation in Drosophila is expressed dynamically in derivatives of all three germ layers. Development 111, 983-992 (1991).

41. Costa, M., Sweeton, D. \& Wieschaus, E. in The Development of Drosophila Melanogaster (eds Bate, M. \& Martinez-Arias, A.) 425-464 (Cold Spring Harbor Laboratory Press, 1993).

42. Kustov, M. et al. Magnetic characterization of micropatterned Nd-Fe-B hard magnetic films using scanning Hall probe microscopy. J. Appl. Phys. 108, 063914 (2010).

43. Wieschaus, E., Sweeton, D. \& Costa, M. in Gastrulation (ed. Keller, R.) 213-223 (Plenum Press, 1991).

44. Farge, E. Mechanical induction of twist in the Drosophila foregut/stomodeal primordium. Curr. Biol. 13, 1365-1377 (2003).

45. Arendt, D. in Gastrulation-From Cells to Embryo (ed. Stern, C. D., Cold Spring Harbor Laboratory press, 2004).

46. Dawes-Hoang, R. E. et al. folded gastrulation, cell shape change and the control of myosin localization. Development 132, 4165-4178 (2005).

47. Seher, T. C., Narasimha, M., Vogelsang, E. \& Leptin, M. Analysis and reconstitution of the genetic cascade controlling early mesoderm morphogenesis in the Drosophila embryo. Mech. Dev. 124, 167-179 (2007).

48. Driquez, B., Bouclet, A. \& Farge, E. Mechanotransduction in mechanically coupled pulsating cells: transition to collective constriction and mesoderm invagination simulation. Phys. Biol. 8, 066007 (2011).

49. Xie, S. \& Martin, A. C. Intracellular signalling and intercellular coupling coordinate heterogeneous contractile events to facilitate tissue folding. Nat. Commun. 6, 7161 (2015).

50. Manning, A. J., Peters, K. A., Peifer, M. \& Rogers, S. L. Regulation of epithelial morphogenesis by the G protein-coupled receptor mist and its ligand fog. Sci. Signal. 6, ra98 (2013).

51. Fernandez-Sanchez, M. E., Brunet, T., Roper, J. C. \& Farge, E. Mechanotransduction's impact in animal development, evolution, and tumorigenesis. Annu. Rev. Cell Dev. Biol. 31, 373-397 (2015).

52. Odell, G. M., Oster, G., Alberch, P. \& Burnside, B. The mechanical basis of morphogenesis. I. Epithelial folding and invagination. Dev. Biol. 85, 446-462 (1981).

53. Morin, X., Daneman, R., Zavortink, M. \& Chia, W. A protein trap strategy to detect GFP-tagged proteins expressed from their endogenous loci in Drosophila. Proc. Natl Acad. Sci. USA 98, 15050-15055 (2001).

54. Weng, M. \& Wieschaus, E. Myosin-dependent remodeling of adherens junctions protects junctions from Snail-dependent disassembly. J. Cell Biol. 212, 219-229 (2016).

55. Roth, S. Mechanisms of dorsal-ventral axis determination in Drosophila embryos revealed by cytoplasmic transplantations. Development 117, 1385-1396 (1993).

56. Xia, L. et al. The niche-dependent feedback loop generates a BMP activity gradient to determine the germline stem cell fate. Curr. Biol. 22, 515-521 (2012).

57. Xu, Z. et al. Impacts of the ubiquitous factor Zelda on Bicoiddependent DNA binding and transcription in Drosophila. Genes Dev. 28, 608621 (2014).

58. Luk, S. K., Kilpatrick, M., Kerr, K. \& Macdonald, P. M. Components acting in localization of bicoid mRNA are conserved among Drosophila species. Genetics 137, 521-530 (1994).

59. Gavis, E. R. \& Lehmann, R. Localization of nanos RNA controls embryonic polarity. Cell 71, 301-313 (1992).

60. Thisse, C., Perrin-Schmitt, F., Stoetzel, C. \& Thisse, B. Sequence-specific transactivation of the Drosophila twist gene by the dorsal gene product. Cell 65, 1191-1201 (1991).

61. Massart, R. Preparation of aqueous magnetic liquids in alkaline and acidic media. IEEE Trans. Magn. 17, 1247-1248 (1981).

62. Skoza, F. C. \& Papahadjopoulos, D. Procedure for preparation of liposomes with large internal aqueous space and high capture by reverse-phase evaporation. Proc. Natl Acad. Sci. USA 75, 4194-4198 (1978).

63. Le Roy, D. et al. Fabrication and characterization of polymer membranes with integrated arrays of high performance micro-magnets. Mater. Today Commun. 6, 50 (2016).

64. Shaw, G., Kramer, R. B. G., Dempsey, N. M. \& Hasselbach, K. et al. A scanning Hall probe microscope for high resolution, large area, variable height magnetic field imaging. Review of Scientific Instruments 87, 113702 (2016). 
65. Desprat, N., Supatto, W., Pouille, P.-A., Beaurepaire, E. \& Farge, E. Tissue deformation modulates twist expression to determine anterior midgut differentiation in Drosophila embryos. Dev. Cell 15, 470-477 (2008).

\section{Acknowledgements}

This research was funded by the ANR (grant \# 11 BSV5014-01), the AVIESAN Cell Biology, Development and Evolution (CNRS, INSERM, INSTITUT CURIE co-funding of the dedicated Magneto-Spinning disk) and the Labex CelTisPhyBio (grant \# 11-LBX0038), and the Labex LANEF (ANR-10-LABX-51-01). We are very grateful to the following members of Institut Néel: Dominique Givord for his input and guidance throughout the work, Klaus Hasselbach for guidance with magnetic field measurements, Jean-Francois Motte for preparing the probes for force measurements and Thibaut Devillers for assistance in micro-magnet fabrication, Vasilis Apostolakeas for assistance in illustrations and to Elena Fernandez-Sanchez from Institut Curie for her contribution to western blots analysis. The micro-patterning of Si substrates for micro-magnet preparation was performed in the PTA-Grenoble cleanroom facility.

\section{Author contributions}

D.M. realized all experiments and B.D. initiated experiments in the posterior endoderm. J.C.-R. realized the westerns. D.L.R., F.D.B. and N.M.D. designed the magnetic tools and D.L.R. fabricated them. G.S. and S.L.D. carried out magnetic field and force measurements, respectively, L.R. carried out field and force simulations. C.M. and A.M. designed and realized the UML. E.F. designed and coordinated the overall work.

\section{Additional information}

Supplementary Information accompanies this paper at http://www.nature.com/ naturecommunications

Competing financial interests: The authors declare no competing financial interests.

Reprints and permission information is available online at http://npg.nature.com/ reprintsandpermissions/

How to cite this article: Mitrossilis, D. et al. Mechanotransductive cascade of Myo-II-dependent mesoderm and endoderm invaginations in embryo gastrulation. Nat. Commun. 8, 13883 doi: 10.1038/ncomms13883 (2017).

Publisher's note: Springer Nature remains neutral with regard to jurisdictional claims in published maps and institutional affiliations.

\section{(c) (i)}

This work is licensed under a Creative Commons Attribution 4.0 International License. The images or other third party material in this article are included in the article's Creative Commons license, unless indicated otherwise in the credit line; if the material is not included under the Creative Commons license, users will need to obtain permission from the license holder to reproduce the material. To view a copy of this license, visit http://creativecommons.org/licenses/by/4.0/

(C) The Author(s) 2017 